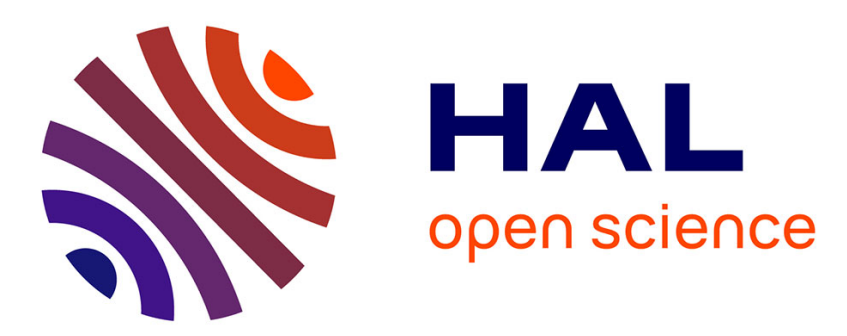

\title{
Minimization of the k-th eigenvalue of the Dirichlet Laplacian
}

Dorin Bucur

\section{To cite this version:}

Dorin Bucur. Minimization of the k-th eigenvalue of the Dirichlet Laplacian. Archive for Rational Mechanics and Analysis, 2012, 206 (3), pp.1073-1083. hal-00956618

\section{HAL Id: hal-00956618 https://hal.science/hal-00956618}

Submitted on 25 Oct 2021

HAL is a multi-disciplinary open access archive for the deposit and dissemination of scientific research documents, whether they are published or not. The documents may come from teaching and research institutions in France or abroad, or from public or private research centers.
L'archive ouverte pluridisciplinaire HAL, est destinée au dépôt et à la diffusion de documents scientifiques de niveau recherche, publiés ou non, émanant des établissements d'enseignement et de recherche français ou étrangers, des laboratoires publics ou privés.

\section{다)(1) $(5$}

Distributed under a Creative Commons Attribution - NonCommerciall 4.0 International 
Version of Record: https://www.sciencedirect.com/science/article/pii/S0022123619301466

Manuscript_1939a5eefb729fa0334d3bec1c5705fc

\title{
MINIMIZATION OF THE $k$-TH EIGENVALUE OF THE ROBIN-LAPLACIAN
}

\author{
DORIN BUCUR AND ALESSANDRO GIACOMINI
}

\begin{abstract}
The paper is concerned with the minimization of the $k$-th eigenvalue of the Laplace operator with Robin boundary conditions, among all open sets of $\mathbb{R}^{N}$ satisfying a volume constraint. We prove the existence of a solution in a relaxed framework and find some qualitative properties of the optimal sets. The main idea is to see these spectral shape optimization questions as free discontinuity problems in the framework of special functions of bounded variation. One of the key difficulties (for $k \geq 3$ ) comes from the fact that the eigenvalues are critical points.
\end{abstract}

KEYWORDS: Robin-Laplacian eigenvalues, shape optimization, relaxation, existence of solutions.

2000 Mathematics Subject Classification: 49J35, 49J45, 26A45, 35R35, 35J20

\section{Contents}

1. Introduction 1

2. Notation and preliminaries 5

2.1. Basic notation 5

2.2. Eigenvalues of the Robin-Laplacian 5

2.3. Functions of bounded variation 6

$\begin{array}{lll}\text { 2.4. A density result for free discontinuity functionals } & 7\end{array}$

$\begin{array}{lll}3 . & \text { The free discontinuity problem } & 7\end{array}$

$\begin{array}{ll}\text { 3.1. The functional space } S B V^{\frac{1}{2}}\left(\mathbb{R}^{N}\right) & 7\end{array}$

3.2. The functional space $S B V_{ \pm}^{\frac{1}{2}}\left(\mathbb{R}^{N} ; \mathbb{R}^{k}\right) \quad 9$

3.3. The free discontinuity functional 12

4. The main result 14

4.1. A density issue 14

4.2. Some technical lemmas 18

4.3. Proof of Theorem $4.1 \quad 24$

5. Further remarks and open questions $\quad 27$

References $\quad 28$

\section{INTRODUCTION}

Given $\Omega \subseteq \mathbb{R}^{N}$ open, bounded and with a Lipschitz boundary, a number $\lambda \in \mathbb{R}$ is said to be an eigenvalue of the Laplace operator under Robin (or Fourier) boundary conditions with constant $\beta>0$ if there exists a nontrivial $u \in W^{1,2}(\Omega)$ such that

$$
\begin{cases}-\Delta u=\lambda u & \text { in } \Omega \\ \frac{\partial u}{\partial \nu}+\beta u=0 & \text { on } \partial \Omega\end{cases}
$$

which in the weak sense means

$$
\forall \varphi \in W^{1,2}(\Omega): \int_{\Omega} \nabla u \cdot \nabla \varphi d x+\beta \int_{\partial \Omega} u \varphi d \mathcal{H}^{N-1}=\lambda \int_{\Omega} u \varphi d x
$$

A.G. is also member of the Gruppo Nazionale per L'Analisi Matematica, la Probabilità e loro Applicazioni (GNAMPA) of the Istituto Nazionale di Alta Matematica (INdAM).

(C) 2019 published by Elsevier. This manuscript is made available under the CC BY NC user license https://creativecommons.org/licenses/by-nc/4.0/ 
Here $\nu$ denotes the outer normal to $\partial \Omega$, while $\mathcal{H}^{N-1}$ stands for the Hausdorff $(N-1)$-dimensional measure on $\mathbb{R}^{N}$, which coincides with the usual area measure on regular hypersurfaces.

It is known that $\Omega$ admits an infinite diverging sequence of eigenvalues

$$
\lambda_{1, \beta}(\Omega)<\lambda_{2, \beta}(\Omega) \leq \lambda_{3, \beta}(\Omega) \leq \cdots \leq \lambda_{k, \beta}(\Omega) \leq \cdots \rightarrow+\infty,
$$

which are given (counting multiplicity) by the min-max formula

$$
\lambda_{k, \beta}(\Omega)=\min _{V \in \mathcal{S}_{k}} \max _{v \in V, v \neq 0} \frac{\int_{\Omega}|\nabla v|^{2} d x+\beta \int_{\partial \Omega} v^{2} d \mathcal{H}^{N-1}}{\int_{\Omega} v^{2} d x},
$$

where $\mathcal{S}_{k}$ denotes the family of vectorial subspaces of $W^{1,2}(\Omega)$ with dimension $k$.

In the present paper we are interested in the shape optimization problem

$$
\inf _{|\Omega|=m} \lambda_{k, \beta}(\Omega),
$$

where $m>0$, and $|\Omega|$ stands for the volume of $\Omega$.

The case $k=1$ is connected to the Faber-Krahn inequality for the Robin-Laplacian, which states that

$$
\lambda_{1, \beta}(\Omega) \geq \lambda_{1, \beta}(B),
$$

where $B$ is a ball such that $|\Omega|=|B|$, with equality if and only if $\Omega$ is itself a ball. The Faber-Krahn inequality was conjectured by PÓLYA in 1951, but it has been established only quite recently by Bossel in 1986 for two dimensional smooth domains [5], and by DANERS in 2006 for $N$-dimensional Lipschitz regular domains [18] (equality in (1.2) has been studied in [19], see also [12]). In terms of the shape optimization problem (1.1), the inequality entails that optimal shapes are balls of volume $m$.

The case $k=2$ has been treated by KENNEDY in [25], where he proved that optimal shapes are given by the union of two disjoint balls of volume $m / 2$.

Existence of optimal shapes is an open problem as soon as $k \geq 3$. In reference [25], it is shown that, in contrast with the cases $k=1,2$, there cannot exist a "universal" optimal shape, that is independent of the value of $\beta$. We refer the reader to reference [4] for a numerical analysis of the optimal shapes, as function of the parameter $\beta$. The numerical evidence leads to conjecture that the minimizing set for (1.1) is the union of $k$ equal disjoint balls provided that $\beta$ is smaller than a critical value depending on $k, m$ and the dimension of the space. On the other hand, when $\beta$ is large, it is expected that the optimal shape will be close to the set minimizing the Dirichlet-Laplacian eigenvalue, under the same volume constraint.

For Dirichlet boundary conditions (which formally corresponds to $\beta=+\infty$ ) the equivalent of problem (1.1) has been quite intensively studied in the last thirty years, starting with the pioneering paper of Buttazzo and Dal Maso [14]. An up to date survey on this issue can be found in the recent book [24]. As soon as $\beta=0$, we deal with the Neumann eigenvalues. In this case, their minimization is not interesting as the minimum of the $k$-th eigenvalue is equal to zero, being achieved on a union of $k+1$ disjoint balls. The challenging problem is related to their maximization, but in this case the nature of the problem is definitely different from (1.1) and the absence of any control of the eigenfunctions at the boundary makes this question to be a very difficult one for arbitrary $k$ (see [24] and [2] for some numerical computations).

In order to study (1.1), a natural step in the Calculus of Variations is to find a relaxation of the problem, that is to extend the problem on a larger class of domains in such a way to prove existence of solutions using the direct method.

The relaxation of spectral shape optimization problems is usually a difficult task. The only general result available in the literature is due to Buttazzo and Dal Maso [14], and deals with spectral problems associated to monotone increasing functions of the Dirichlet-Laplacian eigenvalues. The larger class of domains involved in the relaxation is that of quasi-open sets. The solution of the original problem on Lipschitz domains becomes then an issue of regularity. In this direction, the problem is still unsolved even in the simplest case of the minimization of $k$-th eigenvalue under a volume constraint for $k \geq 3$ : it is known [10] that quasi-open optimal shapes 
are bounded and have finite perimeter, but it is not known even if they are topologically open. Although the Dirichlet boundary conditions are much more studied in the literature (in the framework of free boundary problems), the fact that the $k$-th eigenvalue is itself a critical point, leads to those technical difficulties, not yet overcome. Another difficulty is also related to the kind of constraint we require on the competing domains: if for example the volume constraint is replaced by a perimeter constraint, the situation is completely different, as optimal shapes turn out to be open with fairly smooth boundaries [20].

The optimization of the $k$-th eigenvalue of the Robin-Laplacian (1.1) cannot be relaxed using the Buttazzo-Dal Maso result, basically because the Robin eigenvalues do not enjoy monotonicity properties under inclusion of the domains (see e.g. [23]). By the way, neither the second fundamental requirement in the Buttazzo-Dal Maso theory, namely a good behavior of the spectrum to the so called $\gamma$-convergence, does not occur. In this paper we propose a different kind of relaxation, i.e., we "embed" the shape optimization problem into a free discontinuity problem on a suitable class of functions of bounded variation.

This kind of relaxation has been carried out in the case of the first eigenvalue in [8], where the problem has been dealt with by studying the free discontinuity functional

$$
R_{1, \beta}(u):=\frac{\int_{\mathbb{R}^{N}}|\nabla u|^{2} d x+\beta \int_{J_{u}}\left[\gamma_{1}(u)^{2}+\gamma_{2}(u)^{2}\right] d \mathcal{H}^{N-1}}{\int_{\mathbb{R}^{N}} u^{2} d x},
$$

where $u$ belongs to a suitable space of functions of bounded variation, namely

$$
S B V^{\frac{1}{2}}\left(\mathbb{R}^{N}\right):=\left\{u \in L^{2}\left(\mathbb{R}^{N}\right): u \geq 0, u^{2} \in S B V\left(\mathbb{R}^{N}\right)\right\}
$$

(see Section 2 for a precise definition of the space $S B V$ ). In (1.3), $J_{u}$ stands as usual for the jump set of $u$, while $\gamma_{1}(u)$ and $\gamma_{2}(u)$ denote the two traces of $u$ from both sides of $J_{u}$. The connection of $R_{1, \beta}$ with the first eigenvalue is readily obtained by noticing that if $\psi_{\Omega}$ is the first eigenfunction of $\Omega$, then its extension by zero outside the domain, denoted by $u_{\Omega}:=\psi_{\Omega} 1_{\Omega}$, is such that $\left(J_{u_{\Omega}}=\partial \Omega\right.$ and $\left.\gamma_{2}\left(u_{\Omega}\right)=0\right)$

$$
\lambda_{1, \beta}(\Omega)=R_{1, \beta}\left(u_{\Omega}\right) .
$$

In [8] it is shown that minimizers of $R_{1, \beta}$ on functions whose support has volume $m$ are given by functions of the form

$$
u=\psi_{B} 1_{B},
$$

where $B$ is a ball of volume $m$. In view of (1.4) this entails immediately the Faber-Krahn inequality for the Robin-Laplacian, showing indeed a stronger optimality of the ball, for example among domains with irregular boundary (with suitably defined associated first eigenvalue).

While the analysis of [8] was based on the Bossel-Daners approach (adapted to the free discontinuity setting), the arguments employed in [13] are completely variational, and based on the regularity properties of minimizers of $R_{1, \beta}$. It is shown that the support of minimizers are open bounded set whose topological boundary is rectifiable and with finite area. By means of reflection arguments, it is then proved that they are indeed balls, yielding the Faber-Krahn inequality. Such an analysis is not restricted to the first eigenvalue, but works as well for some semilinear variants such as for example the torsional rigidity (not covered by the Bossel-Daners approach). Moreover it can be employed to find a relaxed class of domains for shape optimization problems involving energies with Robin conditions on the free boundary, as shown in [9]. In this direction, we refer the reader also to $[15,27]$, where, in the context of insulation problems, the regularity of the free boundary of optimal domains has been investigated.

The difficulty to adapt the analysis of $[8,13]$ to problem $(1.1)$ consists in the fact that it is an optimization problem of min-max type. Trying to preserve this structure, we concentrate on the free discontinuity problem

$$
\inf _{|\operatorname{supp}(u)| \leq m} R_{k, \beta}(u)
$$


with

$$
R_{k, \beta}(u):=\max _{v \in V(u)} \frac{\int_{\mathbb{R}^{N}}|\nabla v|^{2} d x+\beta \int_{J_{u}}\left[\gamma_{1}(v)^{2}+\gamma_{2}(v)^{2}\right] d \mathcal{H}^{N-1}}{\int_{\mathbb{R}^{N}} v^{2} d x},
$$

where $u=\left(u_{1}, \ldots, u_{k}\right)$ and $V(u)$ denotes the vectorial space generated by the components of $u$. The function $u$ is now vector valued and belongs to the space (suggested by the case $k=1$ )

$$
\begin{aligned}
S B V_{ \pm}^{\frac{1}{2}}\left(\mathbb{R}^{N} ; \mathbb{R}^{k}\right):=\left\{u=\left(u_{1}, \ldots, u_{k}\right)\right. & : u_{i}^{ \pm} \in S B V^{\frac{1}{2}}\left(\mathbb{R}^{N}\right), \\
& \left.\int_{\mathbb{R}^{N}}|\nabla u|^{2} d x+\int_{J_{u}}\left[\left|\gamma_{1}(u)\right|^{2}+\left|\gamma_{2}(u)\right|^{2}\right] d \mathcal{H}^{N-1}<+\infty\right\},
\end{aligned}
$$

and with $\operatorname{dim}(V(u))=k$. The connection between $R_{k, \beta}$ and the $k$-th eigenvalue of a domain $\Omega$ is pretty close to what was pointed out for $k=1$ : if $\left\{\psi_{\Omega}^{1}, \ldots, \psi_{\Omega}^{k}\right\}$ is the family of the first $k$ eigenfunctions, then

$$
u_{\Omega}:=\left(\psi_{\Omega}^{1} 1_{\Omega}, \ldots, \psi_{\Omega}^{k} 1_{\Omega}\right) \in S B V_{ \pm}^{\frac{1}{2}}\left(\mathbb{R}^{N} ; \mathbb{R}^{k}\right) \quad \text { and } \quad R_{k, \beta}\left(u_{\Omega}\right)=\lambda_{k, \beta}(\Omega) .
$$

It is apparent that the vector valued functions $u$ play for the free discontinuity problem the same role of the vector spaces involved in the min-max formulation of the Robin eigenvalues.

The main results of the paper can be summarized as follows (Theorem 4.1).

(a) The free discontinuity problem (1.5) admits minimizers, which turn out to have a bounded support.

(b) The free discontinuity problem can be considered as a relaxation of the original shape optimization problem thanks to the equality (1.6) and to the relation

$$
\inf _{|\sup (u)| \leq m} R_{k, \beta}(u)=\inf _{|\Omega| \leq m} \lambda_{k, \beta}(\Omega) .
$$

Existence of minimizers follow by direct minimization of the functional $R_{k, \beta}$, thanks to its coercivity and lower semicontinuity properties (see Proposition 3.6 and Proposition 3.12), but it requires a concentration-compactness analysis of inductive type. Fundamental in each step is to prove that the functional admits minimizers with bounded support. This information, trivial for $k=1$ since the support is a ball, follows in general by the analysis of minimizers which arise as limits of a packet of eigenfunctions of a minimizing sequence of domains (see Lemma 4.5 and Theorem 4.6). Coming to (1.7), an immediate observation from (1.6) is that

$$
\inf _{|\operatorname{supp}(u)| \leq m} R_{k, \beta}(u) \leq \inf _{|\Omega| \leq m} \lambda_{k, \beta}(\Omega) .
$$

The converse inequality is proved in Theorem 4.3 and heavily relies on the theory of $S B V$ functions. In a first step we approximate an element $u \in S B V_{ \pm}^{\frac{1}{2}}\left(\mathbb{R}^{N} ; \mathbb{R}^{k}\right)$ by more regular functions (with polyhedral jump sets), following the result of [16]. In a second step, by removing smooth tubular neighbourhoods of the jump set, we recover from the support of $u$ a Lipschitz domain $\Omega$ with a $k$-th eigenvalue lower or comparable to $R_{k, \beta}(u)$.

Given a minimizer of the free discontinuity problem (1.5), we expect its support to provide an optimal shape for the original problem. To recover a classical solution however, we need a regularity analysis which seems very difficult.

A final comment on the choice $\beta>0$. In this case, (1.1) leads to a free discontinuity problem in which the gradient and surface energies are in competition, as they are involved with the same sign. The case $\beta<0$ leads, as for Neumann boundary conditions, to well posed maximization problems for the $k$-th eigenvalue. The nature of the problem changes dramatically, with respect to the minimization problem (1.1). We refer the reader to [3] for a recent collection of results in this direction. A natural relaxation for the problem involves sets with finite perimeter (see [11]).

The paper is organized as follows. After some preliminaries concerning the basic properties of Robin eigenvalues and of functions of bounded variation, in Section 3 we introduce the free discontinuity functional $R_{k, \beta}$, and study its coercivity and lower semicontinuity properties in detail. Section 4 contains the main relaxation result (Theorem 4.1), and is entirely devoted 
to its proof. Subsection 4.1 contains the approximation argument which yields equality (1.7), while in Subsection 4.2 we collect some technical lemmas which are pivotal for the concentrationcompactness argument in the proof of the main result: in particular we treat here the issue of the boundedness of the support of suitable "distinguished" minimizers. The proof of Theorem 4.1 is finally addressed in Subsection 4.3.

\section{Notation AND PRELIMINARIES}

In this section we fix the basic notation employed throughout the paper, and recall some notions concerning the eigenvalues of the Robin-Laplacian operator and the space $B V$ of functions of bounded variation. Moreover we recall a density result for free discontinuity functionals which will be fundamental for our analysis.

2.1. Basic notation. Given $E \subseteq \mathbb{R}^{N}$, we will denote by $|E|$ its Lebesgue measure, by $1_{E}$ its characteristic function, and we set $t E:=\{t x: x \in E\}$ for every $t \in \mathbb{R}$. $\mathcal{H}^{N-1}(E)$ will stand for the Hausdorff $(N-1)$-dimensional measure of $E$ (see [21, Chapter 2]), which coincides with the usual area measure if $E$ is a piecewise regular hypersurface. Two measurable sets $E_{1}, E_{2} \subseteq \mathbb{R}^{N}$ are said to be "well separated" if there exist two open sets $A_{1}, A_{2}$ with $\left|E_{1} \backslash A_{1}\right|=0$ and $\left|E_{2} \backslash A_{2}\right|=0$, and $\operatorname{dist}\left(A_{1}, A_{2}\right)>0$.

For $x \in \mathbb{R}^{N}$ and $r>0, B_{r}(x)$ stands for the ball of center $x$ and radius $r$, while $Q_{r}(x)$ denotes the cube centered at $x$, with sides parallel to the axis of length $r$.

If $\mu$ is a Borel measure on $\mathbb{R}^{N}$ and $A \subseteq \mathbb{R}^{N}$ is Borel regular, we will denote by $\mu\lfloor A$ the restriction of $\mu$ to $A$, and by $|\mu|$ its total variation.

Given a function $u$, we will denote its positive and negative parts with $u^{ \pm}$. If $\Omega \subseteq \mathbb{R}^{N}$ is open and $1 \leq p \leq+\infty, L^{p}\left(\Omega ; \mathbb{R}^{k}\right)$ will stand for the usual space of (classes of) $p$-summable $\mathbb{R}^{k}$-valued functions on $\Omega$, while $W^{k, p}(\Omega)$ will denote the Sobolev space of $p$-summable functions whose derivatives up to order $k$ in the sense of distributions is also $p$-summable.

2.2. Eigenvalues of the Robin-Laplacian. Let $\Omega \subseteq \mathbb{R}^{N}$ be an open bounded set with Lipschitz boundary. Given $\beta>0$, it is known that there exists an infinite sequence

$$
0<\lambda_{1, \beta}(\Omega)<\lambda_{2, \beta}(\Omega) \leq \lambda_{3, \beta}(\Omega) \leq \cdots \leq \lambda_{k, \beta}(\Omega) \leq \cdots \rightarrow+\infty
$$

of values such that for every $k$ there exists $u_{k} \in W^{1,2}(\Omega)$ with $u_{k} \neq 0$ and

$$
\begin{cases}-\Delta u_{k}=\lambda_{k, \beta}(\Omega) u_{k} & \text { in } \Omega \\ \frac{\partial u_{k}}{\partial \nu}+\beta u_{k}=0 & \text { on } \partial \Omega\end{cases}
$$

where $\nu$ denotes the outer normal. The value $\lambda_{k, \beta}(\Omega)$ is said the $k$-th eigenvalue of the Laplace operator under Robin (or Fourier) boundary conditions with coefficient $\beta$.

The eigenvalues can be recovered through the usual Courant-Fisher min-max formula: we have

$$
\lambda_{k, \beta}(\Omega)=\min _{V \in \mathcal{S}_{k}} \max _{v \in V, v \neq 0} \frac{\int_{\Omega}|\nabla v|^{2} d x+\beta \int_{\partial \Omega} v^{2} d \mathcal{H}^{N-1}}{\int_{\Omega} v^{2} d x},
$$

where $\mathcal{S}_{k}$ denotes the family of vectorial subspaces of $W^{1,2}(\Omega)$ with dimension $k$.

The following properties of the Robin eigenvalues will be used frequently throughout the paper.

(a) Scaling: If $t>0$ we have

$$
\lambda_{k, \beta}(t \Omega)=\frac{1}{t^{2}} \lambda_{k, t \beta}(\Omega) .
$$

(b) Faber-Krahn inequality (see $[18,6])$ : We have

$$
\lambda_{1, \beta}(\Omega) \geq \lambda_{1, \beta}(B),
$$

where $B \subseteq \mathbb{R}^{N}$ is a ball such that $|B|=|\Omega|$.

(c) Global estimate for the first eigenvalue of a ball (see e.g. [26, Theorem 4.5]): There exists $C_{N}>0$ such that for every $r>0$

$$
\frac{\beta}{4 r(1+\beta r)} \leq \lambda_{1, \beta}\left(B_{r}\right) \leq \frac{C_{N} \beta}{r(1+\beta r)} .
$$


The properties above will be used throughout the paper. In particular the scaling property (a) is used to establish the key inequality (4.17) in Remark 4.4, while properties (b) and (c) will be fundamental in the proof of Theorem 4.6.

Remark 2.1 (Monotonicity under dilations). The rescaling property (2.2) entails for $t>1$

$$
\lambda_{k, \beta}(t \Omega) \leq \frac{1}{t} \lambda_{k, \beta}(\Omega)<\lambda_{k, \beta}(\Omega),
$$

i.e., we get a monotonicity property under dilation. Notice that in general the eigenvalues of the Robin-Laplacian do not enjoy monotonicity properties under a inclusion of the domains (see e.g. [23]).

2.3. Functions of bounded variation. Let $A \subseteq \mathbb{R}^{N}$ be an open set. We say that $u \in B V(A)$ if $u \in L^{1}(A)$ and its derivative in the sense of distributions is a finite Radon measure on $A$. $B V(A)$ is called the space of functions of bounded variation on $A$. $B V(A)$ is a Banach space under the norm $\|u\|_{B V(A)}:=\|u\|_{L^{1}(A)}+|D u|(A)$. We refer the reader to [1] for an exhaustive treatment of the space $B V$.

Concerning fine properties, a function $u \in B V(A)$ (or better every representative of $u$ ) is a.e. approximately differentiable on $A$, with approximate gradient $\nabla u \in L^{1}\left(A ; \mathbb{R}^{N}\right)$. Moreover, the jump set $J_{u}$ is a $\mathcal{H}^{N-1}$-countably rectifiable set, i.e., $J_{u} \subseteq \cup_{i \in \mathbb{N}} M_{i}$ up to a $\mathcal{H}^{N-1}$-negligible set, with $M_{i}$ a $C^{1}$-hypersurface in $\mathbb{R}^{N}$. The measure $D u$ admits the following representation for every Borel set $B \subseteq A$ :

$$
D u(B)=\int_{B} \nabla u d x+\int_{J_{u} \cap B}\left(\gamma_{2}(u)-\gamma_{1}(u)\right) \nu_{u} d \mathcal{H}^{N-1}+D^{c} u(B),
$$

where $\nu_{u}(x)$ is the normal to $J_{u}$ at $x, \gamma_{1}(u), \gamma_{2}(u)$ are the two traces of $u$ on $J_{u}$ (from the directions $\mp \nu_{u}(x)$ ), and $D^{c} u$ is singular with respect to the Lebesgue measure and concentrated outside $J_{u}$. $D^{c} u$ is usually referred to as the Cantor part of $D u$. The normal $\nu_{u}$ coincides $\mathcal{H}^{N-1}$-a.e. on $J_{u}$ with the normal to the hypersurfaces $M_{i}$. Moreover, $\gamma_{1}(u), \gamma_{2}(u)$ are characterized $\mathcal{H}^{N-1}$-almost everywhere on $J_{u}$ by following Lebesgue-type limit quotient relation

$$
\lim _{r \rightarrow 0} \frac{1}{r^{N}} \int_{B_{r}^{ \pm}(x)}\left|u(x)-\gamma_{2 / 1}(u)(x)\right| d x=0
$$

where $B_{r}^{ \pm}(x):=\left\{y \in B_{r}(x): \nu_{u}(x) \cdot(y-x) \gtrless 0\right\}$ (see [1, Remark 3.79]).

If $A$ is bounded and with a Lipschitz boundary, then $B V(A) \hookrightarrow L^{N / N-1}(A)$. Moreover, the following compactness result holds: if $\left(u_{n}\right)_{n \in \mathbb{N}}$ is bounded in $B V(A)$, there exist $u \in B V(A)$ and a subsequence $\left(u_{n_{k}}\right)_{k \in \mathbb{N}}$ such that

$$
u_{n_{k}} \rightarrow u \quad \text { strongly in } L^{1}(A)
$$

and

$$
D u_{n_{k}} \stackrel{*}{\rightarrow} D u \quad \text { weakly* in the sense of measures. }
$$

We say that $u \in S B V(A)$ if $u \in B V(A)$ and $D^{c} u=0 . S B V(A)$ is called the space of special functions of bounded variation on $A$. This space is very useful to deal with free discontinuity problems in view of the following compactness and lower-semicontinuity result due to L. Ambrosio (see [1, Theorems 4.7-4.8]).

Theorem 2.2. Let $A \subset \mathbb{R}^{N}$ be open and bounded, $\left.p \in\right] 1,+\infty\left[\right.$, and let $\left(u_{n}\right)_{n \in \mathbb{N}}$ be a sequence in $S B V(A)$ such that

$$
\int_{A}\left|\nabla u_{n}\right|^{p} d x+\mathcal{H}^{N-1}\left(J_{u_{n}}\right)+\left\|u_{n}\right\|_{\infty} \leq C
$$

for some $C$ independent of $n$.

Then there exist $u \in S B V(A)$ with $\nabla u \in L^{p}\left(A ; \mathbb{R}^{N}\right)$ and a subsequence $\left(u_{n_{k}}\right)_{k \in \mathbb{N}}$ such that

$$
\begin{gathered}
u_{n_{k}} \rightarrow u \quad \text { strongly in } L^{1}(A), \\
\nabla u_{n_{k}} \rightarrow \nabla u
\end{gathered}
$$


and

$$
\mathcal{H}^{N-1}\left(J_{u}\right) \leq \liminf _{n \rightarrow+\infty} \mathcal{H}^{N-1}\left(J_{u_{n}}\right) .
$$

Remark 2.3. Ambrosio's theorem is still valid if the $L^{\infty}$-bound is replaced by a bound on the total variation (see [7, Theorem 2.3]).

2.4. A density result for free discontinuity functionals. We will make use of a density result in $S B V$ due to Cortesani and Toader [16]. In order to formulate the statement, we will say that $u \in S B V(\Omega)$ with $\Omega$ open set in $\mathbb{R}^{N}$ has polyhedral jumps if $\overline{J_{u}} \cap \Omega$ is the intersection with $\Omega$ of the union of a finite number of $(N-1)$-dimensional simplexes. The density result is the following (see [16, Theorem 3.1]).

Theorem 2.4. Let $\Omega \subseteq \mathbb{R}^{N}$ be open and with Lipschitz boundary, and let $p>1$. Let $u \in$ $S B V(\Omega) \cap L^{\infty}(\Omega)$ be such that $\nabla u \in L^{p}\left(\Omega ; \mathbb{R}^{N}\right)$ and $\mathcal{H}^{N-1}\left(J_{u}\right)<+\infty$.

There exists $\left(u_{n}\right)_{n \in \mathbb{N}}$ such that the following items hold true for every $n \in \mathbb{N}$.

(a) $\mathcal{H}^{N-1}\left(\left(\overline{J_{u_{n}}} \backslash J_{u_{n}}\right) \cap \Omega\right)=0$.

(b) $J_{u_{n}}$ is polyhedral in $\Omega$.

(c) $u_{n} \in W^{k, \infty}\left(\Omega \backslash \overline{J_{u_{n}}}\right)$ for every $k \geq 1$.

Moreover

$$
\begin{aligned}
u_{n} \rightarrow u & \text { strongly in } L^{1}(\Omega), \\
\nabla u_{n} \rightarrow \nabla u & \text { strongly in } L^{p}\left(\Omega ; \mathbb{R}^{N}\right),
\end{aligned}
$$

and

$$
\limsup _{n \rightarrow+\infty} \int_{J_{u_{n}} \cap A} \varphi\left(x, \gamma_{1}\left(u_{n}\right), \gamma_{2}\left(u_{n}\right), \nu_{u_{n}}\right) d \mathcal{H}^{N-1} \leq \int_{J_{u} \cap A} \varphi\left(x, \gamma_{1}(u), \gamma_{2}(u), \nu_{u}\right) d \mathcal{H}^{N-1}
$$

for every open set $A \subset \subset \Omega$ and every upper semicontinuous function $\varphi: \Omega \times \mathbb{R} \times \mathbb{R} \times S^{N-1} \rightarrow$ $\left[0,+\infty\left[\right.\right.$ such that $\varphi(x, a, b, \nu)=\varphi(x, b, a,-\nu)$ for every $x \in \Omega, a, b \in \mathbb{R}$ and $\nu \in S^{N-1}:=\{v \in$ $\left.\mathbb{R}^{N}:|v|=1\right\}$.

\section{The FREE DisCONTINUITY PROBLEM}

In this section we define a free discontinuity functional connected to the min/max formulation of the Robin eigenvalues (2.1), following the ideas developed in [8], where the case of the the first eigenvalue was considered. The functional will be defined in Subsection 3.3, after some preliminaries needed to settle the precise functional framework.

3.1. The functional space $S B V^{\frac{1}{2}}\left(\mathbb{R}^{N}\right)$. We recall here the results contained in [8], where a variational approach to the Faber-Krahn inequality for the Robin-Laplacian, i.e., the optimization of the first eigenvalue, is presented.

We consider the space

$$
S B V^{1 / 2}\left(\mathbb{R}^{N}\right):=\left\{u \in L^{2}\left(\mathbb{R}^{N}\right): u \geq 0 \text { a.e. in } \mathbb{R}^{N} \text { and } u^{2} \in S B V\left(\mathbb{R}^{N}\right)\right\} .
$$

Fine properties of functions in $S B V^{1 / 2}\left(\mathbb{R}^{N}\right)$ are detailed below (see [8, Lemma 1]).

Lemma 3.1. Let $u \in S B V^{\frac{1}{2}}\left(\mathbb{R}^{N}\right)$. Then the following facts hold true.

(a) $u$ is a.e. approximately differentiable (see [1, Definition 3.70]) with approximate gradient $\nabla u$ such that

$$
\nabla\left(u^{2}\right)=2 u \nabla u \quad \text { a.e. in } \mathbb{R}^{N} .
$$

(b) The jump set $J_{u}$ is $\mathcal{H}^{N-1}$-countably rectifiable with normal $\nu_{u}$ such that the jump part of the derivative is given by

$$
D^{j}\left(u^{2}\right)=\left[\gamma_{2}(u)^{2}-\gamma_{1}(u)^{2}\right] \nu_{u} d \mathcal{H}^{N-1}\left\llcorner J_{u} .\right.
$$

(c) For every $\varepsilon>0$ we have $(u-\varepsilon)^{+} \in S B V\left(\mathbb{R}^{N}\right)$.

The main compactness and lower semicontinuity properties of $S B V^{\frac{1}{2}}$ are contained in the following result. For the convenience of the reader, we describe it briefly since it involves arguments that will be used frequently in the rest of the section. For details we refer to [8, Theorem 2]. 
Theorem 3.2. Let $\left(u_{n}\right)_{n \in \mathbb{N}}$ be a sequence in $S B V^{\frac{1}{2}}\left(\mathbb{R}^{N}\right)$ such that

$$
\int_{\mathbb{R}^{N}}\left|\nabla u_{n}\right|^{2} d x+\int_{J_{u_{n}}}\left[\gamma_{1}\left(u_{n}\right)^{2}+\gamma_{2}\left(u_{n}\right)^{2}\right] d \mathcal{H}^{N-1}+\int_{\mathbb{R}^{N}} u_{n}^{2} d x \leq C
$$

for some $C>0$. Then there exist $u \in S B V^{\frac{1}{2}}\left(\mathbb{R}^{N}\right)$ and a subsequence $\left(u_{n_{k}}\right)_{k \in \mathbb{N}}$ such that the following items hold true.

(a) Compactness: $u_{n_{k}} \rightarrow$ strongly in $L_{l o c}^{2}\left(\mathbb{R}^{N}\right)$ and

$$
\nabla u_{n_{k}} \rightarrow \Phi \quad \text { weakly in } L^{2}\left(\mathbb{R}^{N} ; \mathbb{R}^{N}\right)
$$

with

$$
\Phi 1_{s u p p(u)}=\nabla u \text {. }
$$

(b) Lower semicontinuity: for every open set $A \subseteq \mathbb{R}^{N}$ we have

$$
\int_{A}|\nabla u|^{2} d x \leq \liminf _{k \rightarrow+\infty} \int_{A}\left|\nabla u_{n_{k}}\right|^{2} d x
$$

$$
\int_{J_{u} \cap A}^{\text {and }}\left[\gamma_{1}(u)^{2}+\gamma_{2}(u)^{2}\right] d \mathcal{H}^{N-1} \leq \liminf _{k \rightarrow+\infty} \int_{J_{u_{n_{k}}} \cap A}\left[\gamma_{1}\left(u_{n_{k}}\right)^{2}+\gamma_{2}\left(u_{n_{k}}\right)^{2}\right] d \mathcal{H}^{N-1}
$$

Proof. A direct calculation shows that

$$
\left\|u_{n}^{2}\right\|_{B V\left(\mathbb{R}^{N}\right)} \leq \tilde{C}
$$

for some $\tilde{C}>0$. Then there exist a subsequence $\left(u_{n_{k}}\right)_{k \in \mathbb{N}}, u \in L^{2}\left(\mathbb{R}^{N}\right)$ with $u^{2} \in B V\left(\mathbb{R}^{N}\right)$ and $\Phi \in L^{2}\left(\mathbb{R}^{N} ; \mathbb{R}^{N}\right)$ such that

and

$$
u_{n_{k}} \rightarrow u \quad \text { strongly in } L_{l o c}^{2}\left(\mathbb{R}^{N}\right)
$$

$$
\nabla u_{n_{k}} \rightarrow \Phi \quad \text { weakly in } L^{2}\left(\mathbb{R}^{N} ; \mathbb{R}^{N}\right)
$$

Let us fix $\varepsilon>0$. Then we have

$$
\left(u_{n_{k}}-\varepsilon\right)^{+} \in S B V\left(\mathbb{R}^{N}\right),
$$

with

Moreover

$$
\left(u_{n_{k}}-\varepsilon\right)^{+} \rightarrow(u-\varepsilon)^{+} \quad \text { strongly in } L_{l o c}^{2}\left(\mathbb{R}^{N}\right) .
$$

and

$$
\int_{\mathbb{R}^{N}}\left|\nabla\left(u_{n_{k}}-\varepsilon\right)^{+}\right|^{2} d x \leq \int_{\mathbb{R}^{N}}\left|\nabla u_{n_{k}}\right|^{2} d x
$$

$$
\mathcal{H}^{N-1}\left(J_{\left(u_{n_{k}}-\varepsilon\right)^{+}}\right) \leq \frac{1}{\varepsilon^{2}} \int_{J_{u_{n_{k}}}}\left[\gamma_{1}\left(u_{n_{k}}\right)^{2}+\gamma_{2}\left(u_{n_{k}}\right)^{2}\right] d \mathcal{H}^{N-1} .
$$

Finally, $\left(u_{n_{k}}-\varepsilon\right)^{+}$has a uniformly bounded total variation.

By Ambrosio's Theorem 2.2, taking into account Remark 2.3, we deduce that $(u-\varepsilon)^{+} \in$ $S B V\left(\mathbb{R}^{N}\right)$. Moreover

$$
\nabla\left(u_{n_{k}}-\varepsilon\right)^{+} \rightarrow \nabla(u-\varepsilon)^{+} \quad \text { weakly in } L^{2}\left(\mathbb{R}^{N} ; \mathbb{R}^{N}\right)
$$

and for every $A \subseteq \mathbb{R}^{N}$ open, by lower semicontinuity in $S B V$ (see for example [7, Theorem 2.12])

$$
\begin{aligned}
\int_{J_{(u-\varepsilon)+} \cap A}\left[\gamma_{1}\left((u-\varepsilon)^{+}\right)^{2}+\gamma_{2}\left((u-\varepsilon)^{+}\right)^{2}\right] d \mathcal{H}^{N-1} & \\
\leq \liminf _{k \rightarrow+\infty} \int_{J_{\left(u_{n_{k}}-\varepsilon\right)+} \cap A}\left[\gamma_{1}\left(\left(u_{n_{k}}-\varepsilon\right)^{+}\right)^{2}+\gamma_{2}\left(\left(u_{n_{k}}-\varepsilon\right)^{+}\right)^{2}\right] d \mathcal{H}^{N-1} & \quad \leq \liminf _{k \rightarrow+\infty} \int_{J_{u_{n_{k}}} \cap A}\left[\gamma_{1}\left(u_{n_{k}}\right)^{2}+\gamma_{2}\left(u_{n_{k}}\right)^{2}\right] d \mathcal{H}^{N-1}
\end{aligned}
$$


Letting $\varepsilon \rightarrow 0$, we get $u^{2} \in S B V\left(\mathbb{R}^{N}\right)$, and recover inequality (3.6). Since

$$
\nabla\left(u_{n_{k}}-\varepsilon\right)^{+}=\nabla u_{n_{k}} 1_{\left\{u_{n_{k}} \geq \varepsilon\right\}}
$$

we get

$$
\nabla u 1_{\{u \geq \varepsilon\}}=\Phi 1_{\{u \geq \varepsilon\}}
$$

so that we recover relation (3.4) and the lower semicontinuity inequality (3.5).

The main result contained in [8] is the following weak form of the Faber-Krahn inequality for the Robin-Laplacian. It will play an important role it in the proof of Theorem 4.6.

Theorem 3.3 (Faber-Krahn inequality in $S B V^{\frac{1}{2}}\left(\mathbb{R}^{N}\right)$ ). For every $u \in S B V^{\frac{1}{2}}\left(\mathbb{R}^{N}\right)$ we have

$$
\frac{\int_{\mathbb{R}^{N}}|\nabla u|^{2} d x+\beta \int_{J_{u}}\left[\gamma_{1}(u)^{2}+\gamma_{2}(u)^{2}\right] d \mathcal{H}^{N-1}}{\int_{\mathbb{R}^{N}} u^{2} d x} \geq \lambda_{1, \beta}\left(B_{u}\right),
$$

where $B_{u} \subseteq \mathbb{R}^{N}$ is a ball such that $\left|B_{u}\right|=|\operatorname{supp}(u)|$, and $\lambda_{1, \beta}\left(B_{u}\right)$ is the associated first eigenvalue of the Robin-Laplacian.

3.2. The functional space $S B V_{ \pm}^{\frac{1}{2}}\left(\mathbb{R}^{N} ; \mathbb{R}^{k}\right)$. In order to handle the case of higher eigenvalues, and thus in particular to admit functions which change sign, we consider the following space.

Definition 3.4. We say that

$$
u:=\left(u_{1}, \ldots, u_{k}\right) \in S B V_{ \pm}^{\frac{1}{2}}\left(\mathbb{R}^{N} ; \mathbb{R}^{k}\right)
$$

if

and

$$
\left(u_{i}\right)^{ \pm} \in S B V^{\frac{1}{2}}\left(\mathbb{R}^{N}\right) \quad i=1, \ldots, k
$$

$$
\int_{\mathbb{R}^{N}}|\nabla u|^{2} d x+\int_{J_{u}}\left[\left|\gamma_{1}(u)\right|^{2}+\left|\gamma_{2}(u)\right|^{2}\right] d \mathcal{H}^{N-1}<+\infty
$$

where $J_{u}:=J_{u_{1}} \cup \cdots \cup J_{u_{k}}$ and $\gamma_{1 / 2}(u):=\left(\gamma_{1 / 2}\left(u_{1}\right), \ldots, \gamma_{1 / 2}\left(u_{k}\right)\right)$.

In the case $k=1$, we will denote the space simply by $S B V_{ \pm}^{\frac{1}{2}}\left(\mathbb{R}^{N}\right)$.

Remark 3.5. Notice that if $u=\left(u_{1}, \ldots, u_{k}\right) \in S B V_{ \pm}^{\frac{1}{2}}\left(\mathbb{R}^{N} ; \mathbb{R}^{k}\right)$, then in view of Lemma 3.1 , for every $i=1, \ldots, k$ we have that $u_{i}$ is a.e. approximately differentiable with

$$
\nabla u_{i}=\nabla u_{i}^{+}-\nabla u_{i}^{-}
$$

Moreover we have that

is countably $\mathcal{H}^{N-1}$-rectifiable. Since

$$
J_{u_{i}}=J_{u_{i}^{+}} \cup J_{u_{i}^{-}}
$$

$$
\nabla u=\left(\nabla u_{1}, \ldots, \nabla u_{k}\right)
$$

and

$$
J_{u}=J_{u_{1}} \cup \cdots \cup J_{u_{k}} \quad \text { with } \quad \gamma_{i}(u)=\left(\gamma_{i}\left(u_{1}^{+}\right)-\gamma_{i}\left(u_{1}^{-}\right), \ldots, \gamma_{i}\left(u_{k}^{+}\right)-\gamma_{i}\left(u_{k}^{-}\right)\right),
$$

all terms in (3.7) are well defined.

The following compactness and lower semicontinuity result holds true.

Proposition 3.6 (Compactness and lower-semicontinuity in $S B V_{ \pm}^{\frac{1}{2}}\left(\mathbb{R}^{N} ; \mathbb{R}^{k}\right)$ ). Let $\left(u_{n}\right)_{n \in \mathbb{N}}$ be a sequence in $S B V_{ \pm}^{\frac{1}{2}}\left(\mathbb{R}^{N} ; \mathbb{R}^{k}\right)$ such that

$$
\int_{\mathbb{R}^{N}}\left|\nabla u_{n}\right|^{2} d x+\int_{J_{u_{n}}}\left[\left|\gamma_{1}\left(u_{n}\right)\right|^{2}+\left|\gamma_{2}\left(u_{n}\right)\right|^{2}\right] d \mathcal{H}^{N-1}+\int_{\mathbb{R}^{N}}\left|u_{n}\right|^{2} d x \leq C
$$

for some $C>0$. Then there exist $u \in S B V_{ \pm}^{\frac{1}{2}}\left(\mathbb{R}^{N} ; \mathbb{R}^{k}\right), \Phi \in L^{2}\left(\mathbb{R}^{N} ; \mathbb{R}^{k N}\right)$, and a subsequence $\left(u_{n_{h}}\right)_{h \in \mathbb{N}}$ such that

$$
u_{n_{h}} \rightarrow u \quad \text { strongly in } L_{\text {loc }}^{2}\left(\mathbb{R}^{N} ; \mathbb{R}^{k}\right)
$$


and

$$
\nabla u_{n_{h}} \rightarrow \Phi \quad \text { weakly in } L^{2}\left(\mathbb{R}^{N} ; \mathbb{R}^{k N}\right)
$$

with

$$
\Phi 1_{\text {supp }(u)}=\nabla u \text {. }
$$

Moreover, for every open set $A \subseteq \mathbb{R}^{N}$ we have

$$
\int_{A}|\nabla u|^{2} d x \leq \liminf _{h \rightarrow+\infty} \int_{A}\left|\nabla u_{n_{h}}\right|^{2} d x
$$

and

$$
\int_{J_{u} \cap A}\left[\left|\gamma_{1}(u)\right|^{2}+\left|\gamma_{2}(u)\right|^{2}\right] d \mathcal{H}^{N-1} \leq \liminf _{h \rightarrow+\infty} \int_{J_{u_{n_{h}}} \cap A}\left[\left|\gamma_{1}\left(u_{n_{h}}\right)\right|^{2}+\left|\gamma_{2}\left(u_{n_{h}}\right)\right|^{2}\right] d \mathcal{H}^{N-1} .
$$

Proof. If

$$
u_{n}:=\left(u_{1}^{n}, \ldots, u_{k}^{n}\right),
$$

we can apply Theorem 3.2 to the functions $\left(u_{i}^{n}\right)^{ \pm} \in S B V^{\frac{1}{2}}\left(\mathbb{R}^{N}\right)$ for every $i=1, \ldots, k$.

We deduce that we can find a subsequence $\left(u_{n_{h}}\right)_{h \in \mathbb{N}}$ such that for every $i=1, \ldots, k$

$$
\begin{gathered}
u_{i}^{n_{h}} \rightarrow u_{i} \quad \text { strongly in } L_{l o c}^{2}\left(\mathbb{R}^{N}\right), \\
\nabla u_{i}^{n_{h}} \rightarrow \Phi_{i} \quad \text { weakly in } L^{2}\left(\mathbb{R}^{N} ; \mathbb{R}^{N}\right)
\end{gathered}
$$

with $u_{i}^{ \pm} \in S B V^{\frac{1}{2}}\left(\mathbb{R}^{N}\right)$ and $\Phi_{i} 1_{\text {supp }\left(u_{i}\right)}=\nabla u_{i}$. We deduce that (3.9) holds true with $\Phi:=$ $\left(\Phi_{1}, \ldots, \Phi_{k}\right)$. Clearly also (3.10) holds true.

In order to prove (3.11), let us fix $\varepsilon>0$ and consider

$$
u_{n_{h}}^{\varepsilon}:=\left(\left(u_{n_{h}}^{\varepsilon}\right)_{1}, \ldots,\left(u_{n_{h}}^{\varepsilon}\right)_{k}\right)
$$

where

$$
\left(u_{n_{h}}^{\varepsilon}\right)_{i}:=\left(\left(u_{n_{h}}\right)_{i}^{+}-\varepsilon\right)^{+}-\left(\left(u_{n_{h}}\right)_{i}^{-}-\varepsilon\right)^{+} .
$$

Let us denote by $u^{\varepsilon}$ the corresponding function for $u$. By Lemma 3.1 we get $u_{n_{h}}^{\varepsilon} \in S B V\left(\mathbb{R}^{N} ; \mathbb{R}^{k}\right)$, with

$$
u_{n_{h}}^{\varepsilon} \rightarrow u^{\varepsilon} \quad \text { strongly in } L_{l o c}^{2}\left(\mathbb{R}^{N} ; \mathbb{R}^{k}\right) .
$$

Moreover by construction we have

$$
\int_{\mathbb{R}^{N}}\left|\nabla u_{n_{h}}^{\varepsilon}\right|^{2} d x \leq \int_{\mathbb{R}^{N}}\left|\nabla u_{n_{h}}\right|^{2} d x
$$

and

Finally

$$
\int_{J_{u_{n_{h}}^{\varepsilon}}}\left[\left|\gamma_{1}\left(u_{n_{h}}^{\varepsilon}\right)\right|^{2}+\left|\gamma_{2}\left(u_{n_{h}}^{\varepsilon}\right)\right|^{2}\right] d \mathcal{H}^{N-1} \leq \int_{J_{u_{n_{h}}}}\left[\left|\gamma_{1}\left(u_{n_{h}}\right)\right|^{2}+\left|\gamma_{2}\left(u_{n_{h}}\right)\right|^{2}\right] d \mathcal{H}^{N-1}
$$

$$
\mathcal{H}^{N-1}\left(J_{u_{n_{h}}^{\varepsilon}}\right) \leq \frac{1}{\varepsilon^{2}} \int_{J_{u_{n_{h}}}}\left[\left|\gamma_{1}\left(u_{n_{h}}\right)\right|^{2}+\left|\gamma_{2}\left(u_{n_{h}}\right)\right|^{2}\right] d \mathcal{H}^{N-1} \leq C .
$$

As a consequence, by lower semicontinuity in $S B V$ (see for example [7, Theorem 2.12]) we deduce that for every open set $A \subseteq \mathbb{R}^{N}$

$$
\begin{aligned}
\int_{J_{u} \in \cap A}\left[\left|\gamma_{1}\left(u^{\varepsilon}\right)\right|^{2}+\left|\gamma_{2}\left(u^{\varepsilon}\right)\right|^{2}\right] d \mathcal{H}^{N-1} \leq \liminf _{h \rightarrow+\infty} \int_{J_{u_{n}}^{\varepsilon} \cap A}\left[\left|\gamma_{1}\left(u_{n_{h}}^{\varepsilon}\right)\right|^{2}+\left|\gamma_{2}\left(u_{n_{h}}^{\varepsilon}\right)\right|^{2}\right] d \mathcal{H}^{N-1} \\
\quad \leq \liminf _{h \rightarrow+\infty} \int_{J_{u_{n_{h}}} \cap A}\left[\left|\gamma_{1}\left(u_{n_{h}}\right)\right|^{2}+\left|\gamma_{2}\left(u_{n_{h}}\right)\right|^{2}\right] d \mathcal{H}^{N-1}
\end{aligned}
$$

Property (3.11) is recovered by sending $\varepsilon$ to 0 .

Notice finally that

$$
\int_{\mathbb{R}^{N}}|\nabla u|^{2} d x+\int_{J_{u}}\left[\left|\gamma_{1}(u)\right|^{2}+\left|\gamma_{2}(u)\right|^{2}\right] d \mathcal{H}^{N-1}<+\infty,
$$

so that $u \in S B V_{ \pm}^{\frac{1}{2}}\left(\mathbb{R}^{N} ; \mathbb{R}^{k}\right)$, and the proof is concluded. 
Remark 3.7. We will make use of the following strengthened version of the lower semicontinuity inequality (3.11): for every $\varphi \in C_{c}^{\infty}\left(\mathbb{R}^{N}\right)$ with $\varphi \geq 0$

$$
\int_{\mathbb{R}^{N}} \varphi \cdot\left[\left|\gamma_{1}(u)\right|^{2}+\left|\gamma_{2}(u)\right|^{2}\right] d \mathcal{H}^{N-1} \leq \liminf _{h \rightarrow+\infty} \int_{\mathbb{R}^{N}} \varphi \cdot\left[\left|\gamma_{1}\left(u_{n_{h}}\right)\right|^{2}+\left|\gamma_{2}\left(u_{n_{h}}\right)\right|^{2}\right] d \mathcal{H}^{N-1}
$$

This improvement follows by using again [7, Theorem 2.12] to infer

$$
\begin{aligned}
\int_{J_{u^{\varepsilon} \cap A}} \varphi \cdot\left[\left|\gamma_{1}\left(u^{\varepsilon}\right)\right|^{2}+\left|\gamma_{2}\left(u^{\varepsilon}\right)\right|^{2}\right] d \mathcal{H}^{N-1} & \leq \liminf _{h \rightarrow+\infty} \int_{J_{u_{n_{h}}^{\varepsilon} \cap A}} \varphi \cdot\left[\left|\gamma_{1}\left(u_{n_{h}}^{\varepsilon}\right)\right|^{2}+\left|\gamma_{2}\left(u_{n_{h}}^{\varepsilon}\right)\right|^{2}\right] d \mathcal{H}^{N-1} \\
& \leq \liminf _{h \rightarrow+\infty} \int_{J_{u_{n_{h}}} \cap A} \varphi \cdot\left[\left|\gamma_{1}\left(u_{n_{h}}\right)\right|^{2}+\left|\gamma_{2}\left(u_{n_{h}}\right)\right|^{2}\right] d \mathcal{H}^{N-1},
\end{aligned}
$$

and letting $\varepsilon \rightarrow 0$.

When dealing with the free discontinuity functional introduced in the next section, we need to consider linear combinations of the components of functions in $S B V_{ \pm}^{\frac{1}{2}}\left(\mathbb{R}^{N} ; \mathbb{R}^{k}\right)$.

Lemma 3.8. Let $u=\left(u_{1}, \ldots, u_{k}\right) \in S B V_{ \pm}^{\frac{1}{2}}\left(\mathbb{R}^{N} ; \mathbb{R}^{k}\right)$. Then for every $a=\left(a_{1}, \ldots, a_{k}\right) \in \mathbb{R}^{k}$ we have

$$
a_{1} u_{1}+\cdots+a_{k} u_{k} \in S B V_{ \pm}^{\frac{1}{2}}\left(\mathbb{R}^{N}\right) .
$$

Proof. It suffices to check the result for $k=2$ and $a=(1,1)$. Given $u=\left(u_{1}, u_{2}\right)$, let us check that setting

we have

$$
v:=u_{1}+u_{2}
$$

and

$$
v^{ \pm} \in S B V^{\frac{1}{2}}\left(\mathbb{R}^{N}\right)
$$

Let us start with (3.13), dealing for example with the positive part. Let $\varepsilon_{n} \searrow 0$. Since $u_{1}, u_{2} \in$ $S B V_{ \pm}^{\frac{1}{2}}\left(\mathbb{R}^{N}\right)$, we get that

$$
v_{n}:=\left(u_{1}^{+}-\varepsilon_{n}\right)^{+}-\left(u_{1}^{-}-\varepsilon_{n}\right)^{+}+\left(u_{2}^{+}-\varepsilon_{n}\right)^{+}-\left(u_{2}^{-}-\varepsilon_{n}\right)^{+} \in S B V\left(\mathbb{R}^{N}\right) .
$$

As a consequence $v_{n}^{+} \in S B V\left(\mathbb{R}^{N}\right)$ with

$$
v_{n}^{+} \rightarrow v^{+} \quad \text { strongly in } L^{2}\left(\mathbb{R}^{N}\right) \text {. }
$$

Notice that

$$
\left|\nabla v_{n}^{+}\right| \leq\left|\nabla u_{1}\right|+\left|\nabla u_{2}\right|, \quad\left|v_{n}^{+}\right| \leq\left|u_{1}\right|+\left|u_{2}\right| \quad \text { a.e. on } \mathbb{R}^{N}
$$

and that

$$
J_{v_{n}^{+}} \subseteq J_{u_{1}} \cup J_{u_{2}}=J_{u} .
$$

Moreover, on $J_{v_{n}^{+}}$we obtain readily the inequality

$$
\gamma_{1}\left(v_{n}^{+}\right)^{2}+\gamma_{2}\left(v_{n}^{+}\right)^{2} \leq 2\left[\gamma_{1}\left(u_{1}\right)^{2}+\gamma_{2}\left(u_{1}\right)^{2}+\gamma_{1}\left(u_{2}\right)^{2}+\gamma_{2}\left(u_{2}\right)^{2}\right] .
$$

Since $u \in S B V_{ \pm}^{\frac{1}{2}}\left(\mathbb{R}^{N} ; \mathbb{R}^{2}\right)$ entails

$$
\int_{\mathbb{R}^{N}}\left|\nabla u_{1}\right|^{2}+\left|\nabla u_{2}\right|^{2} d x+\int_{J_{u}}\left[\gamma_{1}\left(u_{1}\right)^{2}+\gamma_{1}\left(u_{2}\right)^{2}+\gamma_{2}\left(u_{1}\right)^{2}+\gamma_{2}\left(u_{2}\right)^{2}\right] d \mathcal{H}^{N-1}<+\infty,
$$

in view of $(3.16),(3.17)$ and of the chain rule in $\mathrm{BV}$ (see $\left[1\right.$, Theorem 3.96]), we get that $\left(v_{n}^{+}\right)^{2} \in$ $S B V\left(\mathbb{R}^{N}\right)$, with

$$
\int_{\mathbb{R}^{N}}\left|\nabla v_{n}^{+}\right|^{2} d x+\int_{J_{v_{n}^{+}}}\left[\gamma_{1}\left(v_{n}^{+}\right)^{2}+\gamma_{2}\left(v_{n}^{+}\right)^{2}\right] d \mathcal{H}^{N-1} \leq C
$$

for some $C>0$ independent of $n$. From (3.15) and Theorem 3.2 we thus deduce $v^{+} \in S B V^{\frac{1}{2}}\left(\mathbb{R}^{N}\right)$. 
Inequality (3.14) is then a consequence of (3.18), so that the proof is complete.

3.3. The free discontinuity functional. In order to mimic the $\min / \max$ representation of the Robin-Laplacian eigenvalues (2.1), we consider the following free discontinuity functional.

Definition 3.9 (The space $\mathcal{F}_{k}\left(\mathbb{R}^{N}\right)$ and the functional $\left.R_{k, \beta}\right)$. We say that $u \in \mathcal{F}_{k}\left(\mathbb{R}^{N}\right)$ if

$$
u \in S B V_{ \pm}^{\frac{1}{2}}\left(\mathbb{R}^{N} ; \mathbb{R}^{k}\right) \quad \text { and } \quad \operatorname{dim}(V(u))=k,
$$

where $V(u):=\operatorname{span}\left\{u_{1}, \ldots, u_{k}\right\}$.

Given $\beta>0$ and $u \in \mathcal{F}_{k}\left(\mathbb{R}^{N}\right)$ we set

$$
R_{k, \beta}(u):=\max _{v \in V(u), v \neq 0} \frac{\int_{\mathbb{R}^{N}}|\nabla v|^{2} d x+\int_{J_{u}}\left[\gamma_{1}(v)^{2}+\gamma_{2}(v)^{2}\right] d \mathcal{H}^{N-1}}{\int_{\mathbb{R}^{N}} v^{2} d x} .
$$

Remark 3.10. Some comments are in order.

(a) Thanks to Lemma 3.8 , for $u \in \mathcal{F}_{k}\left(\mathbb{R}^{N}\right)$, we have that

$$
V(u) \subseteq S B V_{ \pm}^{\frac{1}{2}}\left(\mathbb{R}^{N}\right) .
$$

As a consequence the functional $R_{k, \beta}(u)$ is well defined and finite (the jump term is finite thanks to the bound (3.7)).

(b) Notice that the integration of the surface term in $R_{k, \beta}(u)$ takes place on the entire jump set $J_{u}$, so that it may happen that on some of its parts the function $v$ does not admit discontinuities.

Remark 3.11 (Link with the Robin eigenvalue). Let $\Omega \subseteq \mathbb{R}^{N}$ be an open bounded set with Lipschitz boundary, and let $\left\{u_{1}, \ldots, u_{k}\right\}$ denote the first $k$-eigenfunctions of the Robin-Laplacian with constant $\beta$. After an extension by zero outside the domain (still denoted by the same symbol), we get easily

with $J_{u}=\partial \Omega$ and

$$
u:=\left(u_{1}, \ldots, u_{k}\right) \in \mathcal{F}_{k}\left(\mathbb{R}^{N}\right)
$$

$$
R_{k, \beta}(u)=\lambda_{k, \beta}(\Omega) .
$$

We will need the following lower semicontinuity result.

Proposition 3.12 (Lower semicontinuity of $\left.R_{k, \beta}\right)$. Let $\left(u_{n}\right)_{n \in \mathbb{N}}$ be a sequence in $\mathcal{F}_{k}\left(\mathbb{R}^{N}\right)$ such that

for some $u \in \mathcal{F}_{k}\left(\mathbb{R}^{N}\right)$. Then

$$
u_{n} \rightarrow u \quad \text { strongly in } L^{2}\left(\mathbb{R}^{N} ; \mathbb{R}^{k}\right)
$$

$$
R_{k, \beta}(u) \leq \liminf _{n \rightarrow+\infty} R_{k, \beta}\left(u_{n}\right) .
$$

Proof. It is not restrictive to assume that $\left(R_{k, \beta}\left(u_{n}\right)\right)_{n \in \mathbb{N}}$ is bounded. Since every component of $u_{n}$ belongs to $V\left(u_{n}\right)$, we infer immediately that

$$
\int_{\mathbb{R}^{N}}\left|\nabla u_{n}\right|^{2} d x+\int_{J_{u_{n}}}\left[\left|\gamma_{1}\left(u_{n}\right)\right|^{2}+\left|\gamma_{2}\left(u_{n}\right)\right|^{2}\right] d \mathcal{H}^{N-1} \leq C
$$

for some $C>0$.

Let $u=\left(u_{1}, \ldots, u_{k}\right)$ and let us consider

$$
v:=a_{1} u_{1}+\cdots+a_{k} u_{k} \in V(u)
$$

with $a=\left(a_{1}, \ldots, a_{k}\right) \in \mathbb{R}^{k} \backslash\{0\}$, so that $v \neq 0$.

Writing $u_{n}:=\left(u_{1}^{n}, \ldots, u_{k}^{n}\right)$ we get

$$
v_{n}:=a_{1} u_{1}^{n}+\cdots+a_{k} u_{k}^{n} \rightarrow v \quad \text { strongly in } L^{2}\left(\mathbb{R}^{N}\right) .
$$

By Lemma 3.8 we deduce that $v_{n} \in S B V_{ \pm}^{\frac{1}{2}}\left(\mathbb{R}^{N}\right)$ : moreover, thanks to (3.20), we have

$$
\int_{\mathbb{R}^{N}}\left|\nabla v_{n}\right|^{2} d x+\int_{J_{v_{n}}}\left[\gamma_{1}\left(v_{n}\right)^{2}+\gamma_{2}\left(v_{n}\right)^{2}\right] d \mathcal{H}^{N-1} \leq \tilde{C}
$$


for some constant $\tilde{C}$ independent of $n$. In view of Proposition 3.6 we get that

$$
\begin{aligned}
& \int_{\mathbb{R}^{N}}|\nabla v|^{2} d x+\int_{J_{v}}\left[\gamma_{1}(v)^{2}+\gamma_{2}(v)^{2}\right] d \mathcal{H}^{N-1} \\
& \leq \liminf _{n \rightarrow+\infty}\left[\int_{\mathbb{R}^{N}}\left|\nabla v_{n}\right|^{2} d x+\int_{J_{v_{n}}}\left[\gamma_{1}\left(v_{n}\right)^{2}+\gamma_{2}\left(v_{n}\right)^{2}\right] d \mathcal{H}^{N-1}\right]
\end{aligned}
$$

Since $v_{n} \in V\left(u_{n}\right)$ with $v_{n} \neq 0$ if $n$ is large enough, taking into account (3.21) we deduce

$$
\begin{aligned}
& \frac{\int_{\mathbb{R}^{N}}|\nabla v|^{2} d x+\int_{J_{v}}\left[\gamma_{1}(v)^{2}+\gamma_{2}(v)^{2}\right] d \mathcal{H}^{N-1}}{\int_{\mathbb{R}^{N}} v^{2} d x} \\
& \leq \liminf _{n \rightarrow+\infty} \frac{\int_{\mathbb{R}^{N}}\left|\nabla v_{n}\right|^{2} d x+\int_{J_{v_{n}}}\left[\gamma_{1}\left(v_{n}\right)^{2}+\gamma_{2}\left(v_{n}\right)^{2}\right] d \mathcal{H}^{N-1}}{\int_{\mathbb{R}^{N}} v_{n}^{2} d x} \leq \liminf _{n \rightarrow+\infty} R_{k, \beta}\left(u_{n}\right) .
\end{aligned}
$$

Recall that the set $D$ of vectors $a:=\left(a_{1}, \ldots, a_{k}\right) \in \mathbb{R}^{k}$ for which

$$
J_{v}=J_{a_{1} u_{1}+\cdots+a_{k} u_{k}}=J_{u_{1}} \cup J_{u_{2}} \cdots \cup J_{u_{k}}=J_{u} \quad \text { up to } \mathcal{H}^{N-1} \text {-negligible sets }
$$

is dense in $\mathbb{R}^{k}$ (see Remark 3.13 below). Then for such functions $v$ we can replace $J_{v}$ with $J_{u}$ in (3.22) so that by density we infer

$$
\frac{\int_{\mathbb{R}^{N}}|\nabla w|^{2} d x+\int_{J_{u}}\left[\gamma_{1}(w)^{2}+\gamma_{2}(w)^{2}\right] d \mathcal{H}^{N-1}}{\int_{\mathbb{R}^{N}} w^{2} d x} \leq \liminf _{n \rightarrow+\infty} R_{k, \beta}\left(u_{n}\right)
$$

for every $w \in V(u)$. The desired lower semicontinuity result now follows passing to the max on $w$.

Remark 3.13. In the previous proof we used the following property: the set of parameters $a:=\left(a_{1}, \ldots, a_{k}\right) \in \mathbb{R}^{k}$ for which

$$
J_{a_{1} u_{1}+\cdots+a_{k} u_{k}}=J_{u_{1}} \cup J_{u_{2}} \cdots \cup J_{u_{k}}=J_{u} \quad \text { up to } \mathcal{H}^{N-1} \text {-negligible sets }
$$

is dense in $\mathbb{R}^{k}$. The proof that follows employees arguments similar to those of [22, Lemma 3.1] and [17, Lemma 4.5].

Let us consider firstly the case $k=2$. Let $\mathcal{N}$ be the set of vectors $b=\left(b_{1}, b_{2}\right) \in \mathbb{R}^{2}$ such that $J_{b_{1} u_{1}+b_{2} u_{2}}$ is strictly contained in $J_{u}$, that is

$$
\mathcal{H}^{N-1}\left(J_{u} \backslash J_{b_{1} u_{1}+b_{2} u_{2}}\right)>0 .
$$

This means that if we set

$$
C_{b}:=\left\{x \in J_{u}: b_{1} \gamma_{1}\left(u_{1}\right)(x)+b_{2} \gamma_{1}\left(u_{2}\right)(x)=b_{1} \gamma_{2}\left(u_{1}\right)(x)+b_{2} \gamma_{2}\left(u_{2}\right)(x)\right\}
$$

we have $\mathcal{H}^{N-1}\left(C_{b}\right)>0$. If $b^{\prime} \in \mathcal{N}$ is not a multiple of $b$, then we have immediately

$$
\mathcal{H}^{N-1}\left(C_{b^{\prime}} \cap C_{b}\right)=0,
$$

since otherwise on $C_{b^{\prime}} \cap C_{b} \subseteq J_{u}$ we would get $\gamma_{1}\left(u_{1}\right)=\gamma_{2}\left(u_{1}\right)$ and $\gamma_{1}\left(u_{2}\right)=\gamma_{2}\left(u_{2}\right)$. Since $J_{u}$ is $\sigma$-finite with respect to $\mathcal{H}^{N-1}$, we deduce that the set $\mathcal{N}$ is composed, up to a scaling factor, by at most countably many vectors (the family $\left\{C_{b}: b \in \mathcal{N},|b|=1\right\}$ is composed by at most a countable number of sets), so that $\mathbb{R}^{2} \backslash \mathcal{N}$ is dense in $\mathbb{R}^{2}$. This proves our density property.

The case $k>2$ can be treated easily by induction. Given $b \in \mathbb{R}^{k}$, we can find $\left(b_{1}^{\prime}, \ldots, b_{k-1}^{\prime}\right)$ arbitrarily closed to $\left(b_{1}, \ldots, b_{k-1}\right)$ in such a way that

$$
J_{b_{1}^{\prime} u_{1}+\cdots+b_{k-1}^{\prime} u_{k-1}}=J_{u_{1}} \cup \cdots \cup J_{u_{k-1}} \quad \text { up to } \mathcal{H}^{N-1} \text {-negligible sets. }
$$

Setting $v:=b_{1}^{\prime} u_{1}+\cdots+b_{k-1}^{\prime} u_{k-1}$, by the previous step we can find $\left(\alpha, b_{k}^{\prime}\right)$ arbitrarily close to $\left(1, b_{k}\right)$ and such that

$$
J_{\alpha v+b_{k}^{\prime} u_{k}}=J_{v} \cup J_{u_{k}}=J_{u_{1}} \cup \cdots \cup J_{u_{k}}=J_{u} \quad \text { up to } \mathcal{H}^{N-1} \text {-negligible sets. }
$$

Since

$$
\alpha v+\alpha_{k} u_{k}=\alpha b_{1}^{\prime} u_{1}+\cdots+\alpha b_{k-1}^{\prime} u_{k-1}+b_{k}^{\prime} u_{k}
$$


we see that $\left(\alpha b_{1}^{\prime}, \ldots, \alpha b_{k-1}^{\prime}, b_{k}^{\prime}\right)$ can be chosen arbitrarily closed to $\left(b_{1}, \ldots, b_{k}\right)$, so that the conclusion follows.

\section{THE MAIN RESULT}

As mentioned in the Introduction, we are interested in the minimization of the $k$-th eigenvalue of the Robin-Laplace operator with coefficient $\beta>0$ among bounded Lipschitz domains with volume $m>0$. Using the monotonicity of the eigenvalues under dilation, this is equivalent to the shape optimization problem

$$
\inf _{\Omega \in \mathcal{A}_{m}\left(\mathbb{R}^{N}\right)} \lambda_{k, \beta}(\Omega)
$$

where

$$
\mathcal{A}_{m}\left(\mathbb{R}^{N}\right):=\left\{\Omega \subset \mathbb{R}^{N} \text { open, bounded, with Lipschitz boundary and }|\Omega| \leq m\right\} .
$$

Following the considerations of the previous section, in particular in view of Remark 3.11, we are led to consider the free discontinuity problem

$$
\inf _{u \in \mathcal{F}_{k}^{m}\left(\mathbb{R}^{N}\right)} R_{k, \beta}(u)
$$

where

$$
\mathcal{F}_{k}^{m}\left(\mathbb{R}^{N}\right):=\left\{u \in \mathcal{F}_{k}\left(\mathbb{R}^{N}\right):|\operatorname{supp}(u)| \leq m\right\} .
$$

Here $\mathcal{F}_{k}\left(\mathbb{R}^{N}\right)$ and $R_{k, \beta}$ are given in Definition 3.9.

The main result of the paper is the following.

Theorem 4.1 (The main result). For every $k \geq 1$ the free discontinuity problem (4.3) admits a solution with bounded support. Moreover

$$
\min _{u \in \mathcal{F}_{k}^{m}\left(\mathbb{R}^{N}\right)} R_{k, \beta}(u)=\inf _{\Omega \in \mathcal{A}_{m}\left(\mathbb{R}^{N}\right)} \lambda_{k, \beta}(\Omega)=: \lambda_{k, \beta}^{m} .
$$

Remark 4.2. In view of the previous theorem, we can consider the well posed free discontinuity problem (4.3) as a sort of relaxation of the original shape optimization problem (4.1). Intuitively speaking, the optimal shape should be given by the support of a minimizer $u \in \mathcal{F}_{k}\left(\mathbb{R}^{N}\right)$ : to put this intuition on a solid ground, a regularity analysis for the support of minimizers is required. In this direction, we are able at the moment to treat only the case $k=1$ (and some semilinear variants), in which we can show that the support is a ball (see [13]).

The rest of the section is devoted to the proof of Theorem 4.1: in Subsection 4.1 we prove an approximation result which yields the relaxation equality (4.5). In Subsection 4.2 we collect some technical lemmas which are essential to prove the existence of minimizers for problem (4.3), which is addressed in Subsection 4.3 by means of a combination of a concentration-compactness principle and an induction argument, as done in [10].

4.1. A density issue. In Remark 3.11 we pointed out a connection between the free discontinuity functional $R_{k, \beta}$ on the space $\mathcal{F}_{k}\left(\mathbb{R}^{N}\right)$ and the $k$-th eigenvalue of the Robin-Laplacian of a bounded Lipschitz domain. We improve the connection with the following result.

Theorem 4.3 (Density). For every $u \in \mathcal{F}_{k}\left(\mathbb{R}^{N}\right)$ with $|\operatorname{supp}(u)| \leq m$, there exists a sequence $\left(\Omega_{n}\right)_{n \in \mathbb{N}}$ of bounded Lipschitz domains in $\mathbb{R}^{N}$ with $\left|\Omega_{n}\right| \leq m$ and

$$
\limsup _{n \rightarrow+\infty} \lambda_{k, \beta}\left(\Omega_{n}\right) \leq R_{k, \beta}(u) .
$$

Proof. The idea is to approximate $u$ using the Cortesani-Toader regularization given in Theorem 2.4. We divide the proof in several steps.

Step 1. It is not restrictive to assume $u=\left(u_{1}, \ldots, u_{k}\right) \in\left(S B V \cap L^{\infty}\right)\left(\mathbb{R}^{N} ; \mathbb{R}^{k}\right)$ with $\mathcal{H}^{N-1}\left(J_{u}\right)<$ $+\infty$, and with support contained in a compact set.

Indeed it is sufficient to consider for every $i=1, \ldots, k$ and $\varepsilon_{n} \searrow 0$ the functions

$$
\left(u_{i}^{+}-\varepsilon_{n}\right)^{+}-\left(u_{i}^{-}-\varepsilon_{n}\right)^{+}
$$


truncated at levels $\pm n$ and multiplied by smooth cut-off functions between $B_{n}(0)$ and $B_{n+1}(0)$. Let us denote these functions by $u_{i}^{n}$, and let us set

$$
u_{n}:=\left(u_{1}^{n}, \ldots, u_{k}^{n}\right) \text {. }
$$

Clearly $\left|\operatorname{supp}\left(u_{n}\right)\right| \leq m$. By Lemma 3.1 we get $u_{n} \in\left(S B V \cap L^{\infty}\right)\left(\mathbb{R}^{N} ; \mathbb{R}^{k}\right)$, and $\operatorname{dim}\left(V\left(u_{n}\right)\right)=k$ for $n$ large enough, i.e. $u_{n} \in \mathcal{F}_{k}\left(\mathbb{R}^{N}\right)$. In addition

$$
\mathcal{H}^{N-1}\left(J_{u_{n}}\right) \leq \frac{1}{\varepsilon_{n}^{2}} \int_{J_{u}}\left[\left|\gamma_{1}(u)\right|^{2}+\left|\gamma_{2}(u)\right|^{2}\right] d \mathcal{H}^{N-1}<+\infty
$$

The claim follows if we prove that

$$
\lim _{n \rightarrow+\infty} R_{k, \beta}\left(u_{n}\right)=R_{k, \beta}(u)
$$

Notice that

$$
u_{n} \rightarrow u \quad \text { strongly in } L^{2}\left(\mathbb{R}^{N} ; \mathbb{R}^{k}\right)
$$

with

$$
\int_{\mathbb{R}^{N}}\left|\nabla u_{n}\right|^{2} d x+\int_{J_{u_{n}}}\left[\left|\gamma_{1}\left(u_{n}\right)\right|^{2}+\left|\gamma_{2}\left(u_{n}\right)\right|^{2}\right] d \mathcal{H}^{N-1} \leq C
$$

for some $C>0$ independent of $n$. By Proposition 3.12 we get

$$
R_{k, \beta}(u) \leq \liminf _{n \rightarrow+\infty} R_{k, \beta}\left(u_{n}\right)
$$

For every $n$, let

$$
v_{n}:=a_{1}^{n} u_{1}^{n}+\ldots a_{k}^{n} u_{k}^{n} \in V\left(u_{n}\right)
$$

be a function which realizes $R_{k, \beta}\left(u_{n}\right)$. We may assume that up to a subsequence

$$
a_{n}:=\left(a_{1}^{n}, \ldots, a_{k}^{n}\right) \rightarrow a:=\left(a_{1}, \ldots, a_{k}\right)
$$

with $|a|=1$. Then

$$
v_{n} \rightarrow v:=a_{1} u_{1}+\cdots+a_{k} u_{k} \quad \text { strongly in } L^{2}\left(\mathbb{R}^{N}\right)
$$

with

$$
\nabla v_{n} \rightarrow \nabla v \quad \text { strongly in } L^{2}\left(\mathbb{R}^{N} ; \mathbb{R}^{N}\right)
$$

and

$$
\lim _{n \rightarrow+\infty} \int_{J_{u_{n}}}\left[\gamma_{1}\left(v_{n}\right)^{2}+\gamma_{2}\left(v_{n}\right)^{2}\right] d \mathcal{H}^{N-1}=\int_{J_{u}}\left[\gamma_{1}(v)^{2}+\gamma_{2}(v)^{2}\right] d \mathcal{H}^{N-1}
$$

the last relation coming by dominated convergence (recall that $J_{u_{n}} \subseteq J_{u}$ and that $\left|\gamma_{i}\left(u_{n}\right)\right| \leq$ $\left.\left|\gamma_{i}(u)\right|\right)$. We infer

$$
\begin{aligned}
\limsup _{n \rightarrow+\infty} R_{k, \beta}\left(u_{n}\right)=\limsup _{n \rightarrow+\infty} \frac{\int_{\mathbb{R}^{N}}\left|\nabla v_{n}\right|^{2} d x+\int_{J_{u_{n}}}\left[\gamma_{1}\left(v_{n}\right)^{2}+\gamma_{2}\left(v_{n}\right)^{2}\right] d \mathcal{H}^{N-1}}{\int_{\mathbb{R}^{N}} v_{n}^{2} d x} \\
=\frac{\int_{\mathbb{R}^{N}}|\nabla v|^{2} d x+\int_{J_{u}}\left[\gamma_{1}(v)^{2}+\gamma_{2}(v)^{2}\right] d \mathcal{H}^{N-1}}{\int_{\mathbb{R}^{N}} v^{2} d x} \leq R_{k, \beta}(u) .
\end{aligned}
$$

Equation (4.6) follows gathering (4.7) and (4.8), so that the claim of the step follows.

Step 2. Let us consider $u \in\left(S B V \cap L^{\infty}\right)\left(\mathbb{R}^{N} ; \mathbb{R}^{k}\right)$ with $\mathcal{H}^{N-1}\left(J_{u}\right)<+\infty$, supp $(u)$ compactly contained in $B_{R}(0)$ for some $R>0$, and such that $|\operatorname{supp}(u)| \leq m$.

We claim that we can find $w_{n} \in S B V\left(\mathbb{R}^{N} ; \mathbb{R}^{k}\right)$ with $J_{w_{n}}$ polyhedral, $\mathcal{H}^{N-1}\left(\overline{J_{w_{n}}} \backslash J_{w_{n}}\right)=0$, $w_{n} \in\left(W^{1,2} \cap C^{0}\right)\left(\mathbb{R}^{N} \backslash \bar{J}_{w_{n}} ; \mathbb{R}^{k}\right)$,

$$
\overline{\operatorname{supp}\left(w_{n}\right)} \subset \subset B_{R}(0), \quad\left|\overline{\operatorname{supp}\left(w_{n}\right)} \backslash \operatorname{supp}\left(w_{n}\right)\right|=0, \quad \limsup _{n \rightarrow+\infty}\left|\operatorname{supp}\left(w_{n}\right)\right| \leq m,
$$

such that

$$
\begin{gathered}
\nabla w_{n} \rightarrow \nabla u \quad \text { strongly in } L^{2}\left(\mathbb{R}^{N} ; \mathbb{R}^{k N}\right), \\
w_{n} \rightarrow u \quad \text { strongly in } L^{2}\left(\mathbb{R}^{N} ; \mathbb{R}^{k}\right), \\
\limsup _{n \rightarrow+\infty} \mathcal{H}^{N-1}\left(J_{w_{n}}\right) \leq \mathcal{H}^{N-1}\left(J_{u}\right),
\end{gathered}
$$




$$
\limsup _{n \rightarrow+\infty} \int_{J_{w_{n}}}\left[\left|\gamma_{1}\left(w_{n}\right)\right|^{2}+\left|\gamma_{2}\left(w_{n}\right)\right|^{2}\right] d \mathcal{H}^{N-1} \leq \int_{J_{u}}\left[\left|\gamma_{1}(u)\right|^{2}+\left|\gamma_{2}(u)\right|^{2}\right] d \mathcal{H}^{N-1},
$$

and

$$
\begin{aligned}
& \limsup _{n \rightarrow+\infty} \int_{J_{w_{n}}}\left[\gamma _ { 1 } \left(a_{1}\left(w_{n}\right)_{1}\right.\right.\left.\left.+\cdots+a_{k}\left(w_{n}\right)_{k}\right)^{2}+\gamma_{2}\left(a_{1}\left(w_{n}\right)_{1}+\cdots+a_{k}\left(w_{n}\right)_{k}\right)^{2}\right] d \mathcal{H}^{N-1} \\
& \leq \int_{J_{u}}\left[\gamma_{1}\left(a_{1} u_{1}+\cdots+a_{k} u_{k}\right)^{2}+\gamma_{2}\left(a_{1} u_{1}+\cdots+a_{k} u_{k}\right)^{2}\right] d \mathcal{H}^{N-1}
\end{aligned}
$$

Q14a

for every $a=\left(a_{1}, \ldots, a_{k}\right) \in \mathbb{R}^{k}$ with $|a|=1$. Moreover, since the Sobolev 2-capacity of the intersection of simplexes is zero, we may assume that the jump set is composed of a union of disjoint, closed, $(N-1)$ dimensional simplexes.

Indeed, let $\left(v_{h}\right)_{h \in \mathbb{N}}$ be the approximation of $u$ given by Theorem 2.4 in $B_{R}(0)$. If $\eta>0$, let us denote by $u^{\eta}$ the function obtained by replacing each component $u_{i}$ with the function

$$
\left(u_{i}^{+}-\eta\right)^{+}-\left(u_{i}^{-}-\eta\right)^{+}
$$

and let $v_{h}^{\eta}$ denote the function obtained operating in the same way on $v_{h}$. Clearly we have

and

$$
\begin{gathered}
\nabla v_{h}^{\eta} \rightarrow \nabla u^{\eta} \quad \text { strongly in } L^{2}\left(\mathbb{R}^{N} ; \mathbb{R}^{k N}\right), \\
v_{h}^{\eta} \rightarrow u^{\eta} \quad \text { strongly in } L^{2}\left(\mathbb{R}^{N} ; \mathbb{R}^{k}\right),
\end{gathered}
$$

$$
\limsup _{h \rightarrow+\infty} \int_{J_{v_{h}^{\eta}}}\left[\left|\gamma_{1}\left(v_{h}^{\eta}\right)\right|^{2}+\left|\gamma_{2}\left(v_{h}^{\eta}\right)\right|^{2}\right] d \mathcal{H}^{N-1} \leq \int_{J_{u}}\left[\left|\gamma_{1}(u)\right|^{2}+\left|\gamma_{2}(u)\right|^{2}\right] d \mathcal{H}^{N-1} .
$$

The last inequality follow by the corresponding one for $v_{h}$ in view of the choice $\varphi(a, b, \nu)=|a|^{2}+|b|^{2}$ in Theorem 2.4. Moreover, since the choice $\varphi(a, b, \nu) \equiv 1$ yields

$$
\limsup _{h \rightarrow+\infty} \mathcal{H}^{N-1}\left(J_{v_{h}}\right) \leq \mathcal{H}^{N-1}\left(J_{u}\right),
$$

we deduce that for every $a=\left(a_{1}, \ldots, a_{k}\right) \in \mathbb{R}^{k}\left(\operatorname{choose} \varphi(c, d, \nu):=(a \cdot c)^{2}+(a \cdot d)^{2}\right)$

$$
\begin{array}{r}
\limsup _{h \rightarrow+\infty} \int_{J_{v_{h}^{\eta}}}\left[\gamma_{1}\left(a_{1}\left(v_{h}^{\eta}\right)_{1}+\cdots+a_{k}\left(v_{h}^{\eta}\right)_{k}\right)^{2}+\gamma_{2}\left(a_{1}\left(v_{h}^{\eta}\right)_{1}+\cdots+a_{k}\left(v_{h}^{\eta}\right)_{k}\right)^{2}\right] d \mathcal{H}^{N-1} \\
\leq \limsup _{h \rightarrow+\infty} \int_{J_{v_{h}}}\left[\gamma_{1}\left(a_{1}\left(v_{h}\right)_{1}+\cdots+a_{k}\left(v_{h}\right)_{k}\right)^{2}+\gamma_{2}\left(a_{1}\left(v_{h}\right)_{1}+\cdots+a_{k}\left(v_{h}\right)_{k}\right)^{2}\right] d \mathcal{H}^{N-1}+e_{\eta}(a) \\
\quad \leq \int_{J_{u}}\left[\gamma_{1}\left(a_{1} u_{1}+\cdots+a_{k} u_{k}\right)^{2}+\gamma_{2}\left(a_{1} u_{1}+\cdots+a_{k} u_{k}\right)^{2}\right] d \mathcal{H}^{N-1}+e_{\eta}(a),
\end{array}
$$

where $e_{\eta}(a) \rightarrow 0$ as $\eta \rightarrow 0$ uniformly for $a$ varying in compact sets of $\mathbb{R}^{N}$.

Finally notice that

$$
\limsup _{h \rightarrow+\infty}\left|\operatorname{supp}\left(v_{h}^{\eta}\right)\right| \leq m
$$

and that it is not restrictive, multiplying by a suitable cut-off function, to assume that $\operatorname{supp}\left(v_{h}^{\eta}\right)$ is compactly contained in $B_{R}(0)$.

Finally for a.e. $\eta>0$ and for every $h \in \mathbb{N}, i=1, \ldots, k$ we have (the sets are disjoint)

$$
\left|\left\{x \in \mathbb{R}^{N} \backslash \overline{J_{v_{h}}}:\left|\left(v_{h}\right)_{i}(x)\right|=\eta\right\}\right|=0 .
$$

The required approximation is then obtained in the form $w_{n}:=v_{h_{n}}^{\eta_{n}}$, by considering $\eta_{n} \rightarrow 0$ satisfying (4.12), and using a diagonal argument to define $h_{n}$.

Step 3. Let $\left(w_{n}\right)_{n \in \mathbb{N}}$ be given by Step 2. Let us choose $A_{n} \subset \subset B_{R}(0)$ open with smooth boundary, containing $\overline{\operatorname{supp}\left(w_{n}\right)}$, and such that

$$
\limsup _{n \rightarrow+\infty}\left|A_{n}\right| \leq m
$$

This is possible in view of the properties of $\overline{\operatorname{supp}\left(w_{n}\right)}$ given in (4.9). Let us infinitesimally enlarge the disjoint $(N-1)$-simplexes forming $\overline{J_{w_{n}}}$ by considering the closed neighborhood $U_{n}:=\{x \in$ 


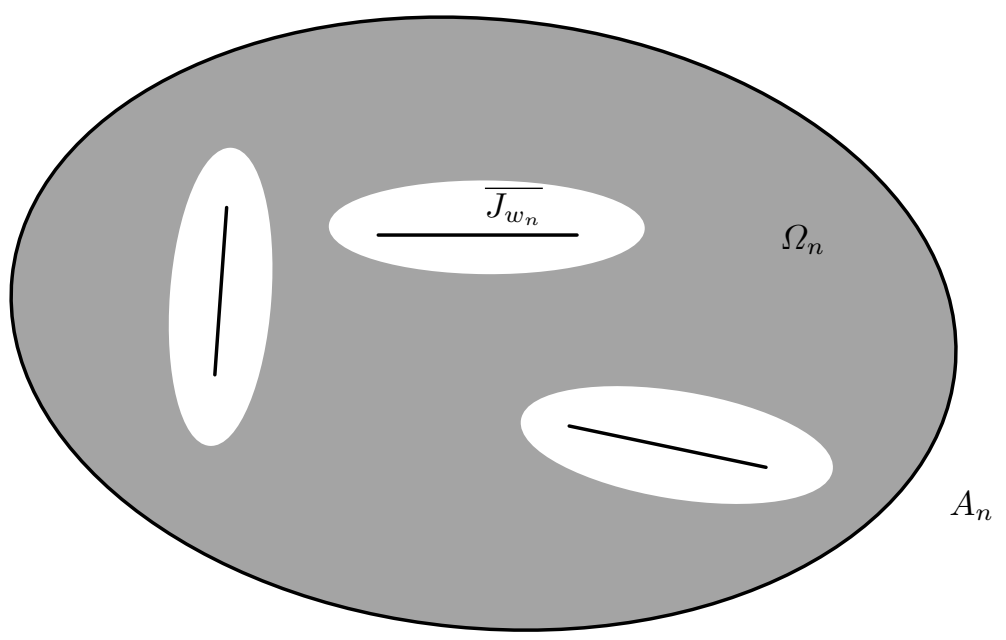

FiguRE 1. The domain $\Omega_{n}$.

$\left.\mathbb{R}^{N}: \operatorname{dist}\left(x, \overline{J_{w_{n}}}\right) \leq \eta_{n}\right\}$ with $\eta_{n} \rightarrow 0$ small enough such that $U_{n} \subset A_{n}$ and $U_{n}$ is Lipschitz. Let us denote $\Omega_{n}:=A_{n} \backslash U_{n}$ (see Figure 1). Then $\Omega_{n}$ is Lipschitz with

$$
\limsup _{n \rightarrow+\infty}\left|\Omega_{n}\right| \leq m \text {. }
$$

Let $u_{n}$ be the restriction of $w_{n}$ to $\Omega_{n}$. We have $u_{n} \in W^{1,2}\left(\Omega_{n}\right)$, and $u_{n}=0$ on $\partial A_{n}$. The enlargement of $\overline{J_{w_{n}}}$ can be chosen so small that, after an extension by zero outside $\Omega_{n}$ (still denoted by the same symbol)

$$
\begin{gathered}
\nabla u_{n} \rightarrow \nabla u \quad \text { strongly in } L^{2}\left(\mathbb{R}^{N} ; \mathbb{R}^{k N}\right), \\
u_{n} \rightarrow u \quad \text { strongly in } L^{2}\left(\mathbb{R}^{N} ; \mathbb{R}^{k}\right),
\end{gathered}
$$

and, in view of (4.10) and (4.11), also such that

$$
\limsup _{n \rightarrow+\infty} \int_{\partial \Omega_{n}}\left|u_{n}\right|^{2} d \mathcal{H}^{N-1} \leq \int_{J_{u}}\left[\left|\gamma_{1}(u)\right|^{2}+\left|\gamma_{2}(u)\right|^{2}\right] d \mathcal{H}^{N-1}
$$

and

$$
\begin{aligned}
\limsup _{n \rightarrow+\infty} \int_{\partial \Omega_{n}}\left(a_{1}\left(u_{n}\right)_{1}+\right. & \left.\cdots+a_{k}\left(u_{n}\right)_{k}\right)^{2} d \mathcal{H}^{N-1} \\
& \leq \int_{J_{u}}\left[\gamma_{1}\left(a_{1} u_{1}+\cdots+a_{k} u_{k}\right)^{2}+\gamma_{2}\left(a_{1} u_{1}+\cdots+a_{k} u_{k}\right)^{2}\right] d \mathcal{H}^{N-1}
\end{aligned}
$$

for every $a \in \mathbb{R}^{k}$ with $|a|=1$.

Notice that for $n$ large enough, $\left\{\left(u_{n}\right)_{1}, \ldots,\left(u_{n}\right)_{k}\right\}$ are linearly independent. Let $a^{n}:=\left(a_{1}^{n}, \ldots, a_{k}^{n}\right)$ with $\left|a^{n}\right|=1$ be such that

is achieved on

$$
\max _{v \in \operatorname{span}\left\{\left(u_{n}\right)_{1}, \ldots,\left(u_{n}\right)_{k}\right\}} \frac{\int_{\Omega_{n}}|\nabla v|^{2} d x+\beta \int_{\partial \Omega_{n}} v^{2} d \mathcal{H}^{N-1}}{\int_{\Omega_{n}} v^{2} d x}
$$

$$
v_{n}:=a_{1}^{n}\left(u_{n}\right)_{1}+\cdots+a_{k}^{n}\left(u_{n}\right)_{k} \in W^{1,2}\left(\Omega_{n}\right) .
$$


We may assume up to a subsequence that

$$
a^{n} \rightarrow a:=\left(a_{1}, \ldots, a_{k}\right) .
$$

Then we have, by considering $\tilde{v}_{n}:=a_{1}\left(u_{n}\right)_{1}+\cdots+a_{k}\left(u_{n}\right)_{k}$ and taking into account (4.14) and (4.15)

$$
\begin{aligned}
\limsup _{n \rightarrow+\infty} \lambda_{k, \beta}\left(\Omega_{n}\right) \leq \limsup _{n \rightarrow+\infty} \frac{\int_{\Omega_{n}}\left|\nabla v_{n}\right|^{2} d x+\beta \int_{\partial \Omega_{n}} v_{n}^{2} d \mathcal{H}^{N-1}}{} & \int_{\Omega_{n}} v_{n}^{2} d x \\
= & \limsup _{n \rightarrow+\infty} \frac{\int_{\Omega_{n}}\left|\nabla \tilde{v}_{n}\right|^{2} d x+\beta \int_{\partial \Omega_{n}} \tilde{v}_{n}^{2} d \mathcal{H}^{N-1}}{\int_{\Omega_{n}} \tilde{v}_{n}^{2} d x} \leq R_{k, \beta}(u) .
\end{aligned}
$$

Thanks to (4.13), the conclusion follows up to a small dilation (see (2.2)), if necessary, to match the constraint $\left|\Omega_{n}\right| \leq m$.

Q15 i Remark 4.4. The approximation result of Theorem 4.3 and Remark 3.11 entail immediately the validity of equality (4.5). In view of the rescaling property (2.2), we get also that for every $t>0$

$$
\inf _{u \in \mathcal{F}_{k}^{t^{N}}\left(\mathbb{R}^{N}\right)} R_{k, \beta}(u)=\frac{1}{t^{2}} \inf _{u \in \mathcal{F}_{k}^{m}\left(\mathbb{R}^{N}\right)} R_{k, t \beta}(u) .
$$

Q15 ii

Finally, using the monotonicity property (2.4), we deduce that for every $t>1$

$$
\lambda_{k, \beta}^{m t^{N}} \leq \frac{1}{t} \lambda_{k, \beta}^{m}<\lambda_{k, \beta}^{m}
$$

The last strict inequality is a consequence of the fact that for every $\beta, m>0$ we have $\lambda_{k, \beta}^{m}>0$. We conclude that for every $t>1$

$$
\lambda_{k, \beta}^{m t}<\lambda_{k, \beta}^{m}
$$

This inequality will be used in the proof of Theorem 4.1.

4.2. Some technical lemmas. In this subsection we collect some technical lemmas which will be pivotal in the proof of the existence of minimizers of the free discontinuity problem (4.3), based on a concentration compactness principle. Lemma 4.5 and Theorem 4.6 will be used to deal with the so called "compact" case, while Lemma 4.7 will be employed in the "dichotomy" case.

Lemma 4.5 (Distinguished minimizers). Let $\left(\Omega_{n}\right)_{n \in \mathbb{N}}$ be a sequence of Lipschitz domains with $\left|\Omega_{n}\right|=m$ and

$$
\lambda_{k, \beta}\left(\Omega_{n}\right) \rightarrow \lambda_{k, \beta}^{m}=\inf _{u \in \mathcal{F}_{k}^{m}\left(\mathbb{R}^{N}\right)} R_{k, \beta}(u) .
$$

Let $\left\{u_{1}^{n}, \ldots, u_{k}^{n}\right\}$ denote the first $k$ eigenfunctions of the Robin-Laplacian for $\Omega_{n}$, which we assume, as usual, to form an orthonormal system in $L^{2}\left(\Omega_{n}\right)$.

After an extension by zero outside $\Omega_{n}$, still denoted by the same symbol, we have

$$
u_{n}:=\left(u_{1}^{n}, \ldots, u_{k}^{n}\right) \in \mathcal{F}_{k}^{m}\left(\mathbb{R}^{N}\right),
$$

and the following items hold true.

(a) $\left(u_{n}\right)_{n \in \mathbb{N}}$ is a minimizing sequence for problem (4.3).

(b) Assume that

$$
u_{n} \rightarrow u:=\left(u_{1}, \ldots, u_{k}\right) \quad \text { strongly in } L^{2}\left(\mathbb{R}^{N} ; \mathbb{R}^{k}\right) .
$$

Then $\left\{u_{1}, \ldots, u_{k}\right\}$ forms an orthonormal system in $L^{2}\left(\mathbb{R}^{N}\right)$, and $u$ is a minimizer of problem (4.3) such that for every $i=1, \ldots, k$ and $\varphi \in C_{c}^{\infty}\left(\mathbb{R}^{N}\right)$ with $\varphi \geq 0$

$$
\int_{\mathbb{R}^{N}} \nabla u_{i} \cdot \nabla\left(\varphi u_{i}\right) d x+\beta \int_{J_{u_{i}}} \varphi \cdot\left[\gamma_{1}\left(u_{i}\right)^{2}+\gamma_{2}\left(u_{i}\right)^{2}\right] d \mathcal{H}^{N-1} \leq \lambda_{k, \beta}^{m} \int_{\mathbb{R}^{N}} \varphi u_{i}^{2} d x
$$


Proof. The fact that $u_{n} \in \mathcal{F}_{k}^{m}\left(\mathbb{R}^{N}\right)$ together with point (a) follow from Remark 3.11 in view of equation (4.18). In particular we have for every $n \in \mathbb{N}$ (recall that $J_{u_{n}}=\partial \Omega$ since $u_{n}=0$ outside $\left.\Omega_{n}\right)$

$$
\int_{\mathbb{R}^{N}}\left|\nabla u_{n}\right|^{2} d x+\beta \int_{J_{u_{n}}}\left[\left|\gamma_{1}\left(u_{n}\right)\right|^{2}+\left|\gamma_{2}\left(u_{n}\right)\right|^{2}\right] d \mathcal{H}^{N-1} \leq k \lambda_{k, \beta}\left(\Omega_{n}\right) .
$$

Let us come to point (b). Thanks to (4.20), by Proposition 3.6 we infer

$$
u \in S B V_{ \pm}^{\frac{1}{2}}\left(\mathbb{R}^{N} ; \mathbb{R}^{k}\right)
$$

Moreover $|\operatorname{supp}(u)| \leq m$, and $\operatorname{dim}(V(u))=k$, as $\left\{u_{1}, \ldots, u_{k}\right\}$ is an orthonormal system in $L^{2}\left(\mathbb{R}^{N}\right)$. We thus conclude that $u \in \mathcal{F}_{k}^{m}\left(\mathbb{R}^{N}\right)$. Moreover $u$ is a minimizer of problem (4.3) thanks to the lower semicontinuity property of $R_{k, \beta}$ given in Proposition 3.12.

Let us prove inequality (4.19). Let $\varphi \in C_{c}^{\infty}\left(\mathbb{R}^{N}\right)$ with $\varphi \geq 0$. Since $u_{i}^{n}$ is an eigenfunction for $\Omega_{n}$ we may write

$$
\int_{\Omega_{n}} \nabla u_{i}^{n} \cdot \nabla\left(\varphi \cdot u_{i}^{n}\right) d x+\beta \int_{\partial \Omega_{n}} \varphi \cdot\left(u_{i}^{n}\right)^{2} d \mathcal{H}^{N-1}=\lambda_{i, \beta}\left(\Omega_{n}\right) \int_{\Omega_{n}} \varphi \cdot\left(u_{i}^{n}\right)^{2} d x .
$$

After the extension to zero outside $\Omega_{n}$ we get

$$
\int_{\mathbb{R}^{N}} \nabla u_{i}^{n} \cdot \nabla\left(\varphi \cdot u_{i}^{n}\right) d x+\beta \int_{J_{u_{i}^{n}}} \varphi \cdot\left[\gamma_{1}\left(u_{i}^{n}\right)^{2}+\gamma_{2}\left(u_{i}^{n}\right)^{2}\right] d \mathcal{H}^{N-1}=\lambda_{i, \beta}\left(\Omega_{n}\right) \int_{\mathbb{R}^{N}} \varphi \cdot\left(u_{i}^{n}\right)^{2} d x
$$

We know that, up to a subsequence,

$$
\nabla u_{i}^{n} \rightarrow \Phi^{i} \quad \text { weakly in } L^{2}\left(\mathbb{R}^{N} ; \mathbb{R}^{N}\right)
$$

with $\Phi^{i} 1_{\text {supp }\left(u_{i}\right)}=\nabla u_{i}$. This is a consequence of (4.20) and the strong convergence in $L^{2}$. By expanding the first term in (4.21) as

$$
\int_{\mathbb{R}^{N}} \nabla u_{i}^{n} \cdot \nabla\left(\varphi \cdot u_{i}^{n}\right) d x=\int_{\mathbb{R}^{N}} u_{i}^{n} \nabla u_{i}^{n} \cdot \nabla \varphi d x+\int_{\mathbb{R}^{N}} \varphi \cdot\left|\nabla u_{i}^{n}\right|^{2} d x
$$

in view of the strong convergence of $u_{n}$ and since $\varphi \geq 0$, we obtain

$$
\begin{aligned}
& \liminf _{n \rightarrow+\infty} \int_{\mathbb{R}^{N}} \nabla u_{i}^{n} \cdot \nabla\left(\varphi u_{i}^{n}\right) d x \geq \int_{\mathbb{R}^{N}} u_{i} \Phi^{i} \cdot \nabla \varphi d x+\int_{\mathbb{R}^{N}} \varphi \cdot\left|\Phi^{i}\right|^{2} d x \\
& \geq \int_{\mathbb{R}^{N}} u_{i} \nabla u_{i} \cdot \nabla \varphi d x+\int_{\mathbb{R}^{N}} \varphi \cdot\left|\nabla u_{i}\right|^{2} d x=\int_{\mathbb{R}^{N}} \nabla u_{i} \cdot \nabla\left(\varphi u_{i}\right) d x .
\end{aligned}
$$

Moreover by Remark 3.7 we get also

$$
\liminf _{n \rightarrow+\infty} \int_{J_{u_{i}^{n}}^{n}} \varphi \cdot\left[\gamma_{1}\left(u_{i}^{n}\right)^{2}+\gamma_{2}\left(u_{i}^{n}\right)^{2}\right] d \mathcal{H}^{N-1} \geq \int_{J_{u_{i}}} \varphi \cdot\left[\gamma_{1}\left(u_{i}\right)^{2}+\gamma_{2}\left(u_{i}\right)^{2}\right] d \mathcal{H}^{N-1} .
$$

Finally since

$$
\lambda_{i, \beta}\left(\Omega_{n}\right) \leq \lambda_{k, \beta}\left(\Omega_{n}\right)=R_{k, \beta}\left(u_{n}\right) \rightarrow \lambda_{k, \beta}^{m},
$$

we infer

$$
\limsup _{n \rightarrow+\infty} \lambda_{i, \beta}\left(\Omega_{n}\right) \int_{\mathbb{R}^{N}} \varphi \cdot\left(u_{i}^{n}\right)^{2} d x \leq \lambda_{k, \beta}^{m} \int_{\mathbb{R}^{N}} \varphi \cdot u_{i}^{2} d x .
$$

Collecting (4.22), (4.23) and (4.24), we deduce that inequality (4.19) holds true, so that point (b) follows, and the proof is concluded.

Theorem 4.6 (Boundedness of the support of distinguished minimizers). Let $u \in$ $\mathcal{F}_{k}^{m}\left(\mathbb{R}^{N}\right)$ be a minimizer of problem (4.3) satisfying point (b) of Lemma 4.5. Then supp $(u)$ is bounded. 
Proof. Assume by contradiction that $\operatorname{supp}(u)$ is unbounded. Up to a rotation and a translation, it is not restrictive to assume that

$$
\operatorname{supp}(u) \cap\left\{x_{1}>t\right\}
$$

has positive volume for every $t \geq 0$. We divide the proof in several steps.

Step 1. For a.e. $t \in \mathbb{R}$ large enough we have $u 1_{\left\{x_{1}<t\right\}} \in \mathcal{F}_{k}^{m}\left(\mathbb{R}^{N}\right)$. We claim that for a.e. $t \geq 0$ large enough

$$
R_{k, \beta}\left(u 1_{\left\{x_{1}<t\right\}}\right) \leq R_{k, \beta}(u)+\frac{2 \beta \delta(t)}{c_{2}(t)},
$$

where

$$
\delta(t):=\int_{\left\{x_{1}=t\right\}}|\nabla u|^{2} d \mathcal{H}^{N-1}+\beta \sum_{i=1}^{k} \int_{J_{u_{i}} \cap\left\{x_{1}=t\right\}}\left[\gamma_{1}\left(u_{i}\right)^{2}+\gamma_{2}\left(u_{i}\right)^{2}\right] d \mathcal{H}^{N-2}
$$

and

$$
c_{2}(t)=\lambda_{1, \beta}\left(B^{\prime}(t)\right)
$$

where $B^{\prime}(t) \subset \mathbb{R}^{N-1}$ is a ball such that

$$
\left|B^{\prime}(t)\right|=\mathcal{H}^{N-1}\left(\operatorname{supp}(u) \cap\left\{x_{1}=t\right\}\right)>0 .
$$

In order to prove the claim, let us write the quantity $R_{k, \beta}\left(u 1_{\left\{x_{1}<t\right\}}\right)$ as (notice that $\left\{x_{1}=t\right\}$ is now a possible jump set for $u 1_{\left\{x_{1}<t\right\}}$ )

$$
\frac{\int_{\left\{x_{1}<t\right\}}|\nabla U(t)|^{2} d x+\beta \int_{J_{u} \cap\left\{x_{1}<t\right\}}\left[\gamma_{1}(U(t))^{2}+\gamma_{2}(U(t))^{2}\right] d \mathcal{H}^{N-1}+\beta \int_{\left\{x_{1}=t\right\}} U(t)^{2} d \mathcal{H}^{N-1}}{\int_{\left\{x_{1}<t\right\}} U^{2}(t) d x},
$$

where

$$
U(t):=\sum_{i=1}^{k} a_{i}(t) u_{i} \in S B V_{ \pm}^{\frac{1}{2}}\left(\mathbb{R}^{N}\right) \quad \text { with } \quad \sum_{i=1}^{k} a_{i}(t)^{2}=1
$$

is such that $U(t) 1_{\left\{x_{1}<t\right\}}$ realizes $R_{k, \beta}\left(u 1_{\left\{x_{1}<t\right\}}\right)$. Since $\left\{u_{1}, \ldots, u_{k}\right\}$ forms an orthonormal system in $L^{2}\left(\mathbb{R}^{N}\right)$ we have

so that

$$
\int_{\left\{x_{1}<t\right\}} U(t)^{2} d x=1-\int_{\left\{x_{1}>t\right\}} U(t)^{2} d x
$$

$$
\begin{aligned}
R_{k, \beta}\left(u 1_{\left\{x_{1}<t\right\}}\right) \leq & \frac{1}{1-\int_{\left\{x_{1}>t\right\}} U^{2}(t) d x}\left[R_{k, \beta}(u)+\beta \int_{\left\{x_{1}=t\right\}} U^{2}(t) d \mathcal{H}^{N-1}\right. \\
& \left.-\int_{\left\{x_{1}>t\right\}}|\nabla U(t)|^{2} d x-\beta \int_{J_{u} \cap\left\{x_{1}>t\right\}}\left[\gamma_{1}(U(t))^{2}+\gamma_{2}(U(t))^{2}\right] d \mathcal{H}^{N-1}\right]
\end{aligned}
$$

We have

$$
\int_{\left\{x_{1}>t\right\}} U^{2}(t) d x \leq \sum_{i=1}^{k} \int_{\left\{x_{1}>t\right\}} u_{i}^{2} d x \stackrel{t \rightarrow+\infty}{\longrightarrow} 0 .
$$

The last equality can be seen as a consequence of the dominated convergence theorem. Thus we can write for $t$ large enough

$$
\begin{aligned}
R_{k, \beta}\left(u 1_{\left\{x_{1}<t\right\}}\right) \leq & \left(R_{k, \beta}(u)+\beta \int_{\left\{x_{1}=t\right\}} U^{2}(t) d \mathcal{H}^{N-1}-\int_{\left\{x_{1}>t\right\}}|\nabla U(t)|^{2} d x\right. \\
& \left.-\beta \int_{J_{u} \cap\left\{x_{1}>t\right\}}\left[\gamma_{1}(U(t))^{2}+\gamma_{2}(U(t))^{2}\right] d \mathcal{H}^{N-1}\right) \cdot\left(1+2 \int_{\left\{x_{1}>t\right\}} U(t)^{2} d x\right),
\end{aligned}
$$


$R_{k, \beta}\left(u 1_{\left\{x_{1}<t\right\}}\right) \geq 0$. Finally,

$$
\begin{aligned}
R_{k, \beta}\left(u 1_{\left\{x_{1}<t\right\}}\right) \leq R_{k, \beta}(u) & +2 R_{k, \beta}(u) \int_{\left\{x_{1}>t\right\}} U(t)^{2} d x+2 \beta \int_{\left\{x_{1}=t\right\}} U(t)^{2} d \mathcal{H}^{N-1} \\
& -\int_{\left\{x_{1}>t\right\}}|\nabla U(t)|^{2} d x-\beta \int_{J_{u} \cap\left\{x_{1}>t\right\}}\left[\gamma_{1}(U(t))^{2}+\gamma_{2}(U(t))^{2}\right] d \mathcal{H}^{N-1} .
\end{aligned}
$$

If we reflect the function $U(t) 1_{\left\{x_{1}>t\right\}}$ across the hyperplane $\left\{x_{1}=t\right\}$, we obtain a new function $v(t) \in S B V_{ \pm}^{\frac{1}{2}}\left(\mathbb{R}^{N}\right)$. We can thus use the Faber-Krahn inequality of Theorem 3.3 for the positive and negative parts of $v(t)$ and obtain (in terms of $U(t)$ )

$$
\int_{\left\{x_{1}>t\right\}}|\nabla U(t)|^{2} d x+\beta \int_{J_{u} \cap\left\{x_{1}>t\right\}}\left[\gamma_{1}(U(t))^{2}+\gamma_{2}(U(t))^{2}\right] d \mathcal{H}^{N-1} \geq c_{1}(t) \int_{\left\{x_{1}>t\right\}} U(t)^{2} d x
$$

with

$$
c_{1}(t)=\lambda_{1, \beta}(B(t))
$$

where $B(t) \subseteq \mathbb{R}^{N}$ is a ball such that

$$
|B(t)|=2\left|\operatorname{supp}(U(t)) \cap\left\{x_{1}>t\right\}\right| \leq 2\left|\operatorname{supp}(u) \cap\left\{x_{1}>t\right\}\right|
$$

Notice that $c_{1}(t) \rightarrow+\infty$ as $t \rightarrow+\infty$ since $|B(t)| \rightarrow 0$ (see (2.3)). We conclude that for $t$ large enough

$$
R_{k, \beta}\left(u 1_{\left\{x_{1}<t\right\}}\right) \leq R_{k, \beta}(u)+2 \beta \int_{\left\{x_{1}=t\right\}} U(t)^{2} d \mathcal{H}^{N-1} \leq R_{k, \beta}(u)+2 \beta \sum_{i=1}^{k} \int_{\left\{x_{1}=t\right\}} u_{i}^{2} d \mathcal{H}^{N-1}
$$

Note that the above inequality entails immediately that $\mathcal{H}^{N-1}\left(\operatorname{supp}(u) \cap\left\{x_{1}=t\right\}\right)>0$, otherwise it would be against the strict inequality (4.17), since $\left|\operatorname{supp}(u) \cap\left\{x_{1}>t\right\}\right|>0$.

In view of the theory of sections for BV functions (see [1, Section 3.11]), we have that for a.e. $t \in \mathbb{R}$ the section of $u_{i}$ on the hyperplane $\left\{x_{1}=t\right\}$ belongs to $S B V_{ \pm}^{\frac{1}{2}}\left(\mathbb{R}^{N-1}\right)$, with associated jump set given by $J_{u_{i}} \cap\left\{x_{1}=t\right\}$, and pair of traces given again by $\left(\gamma_{1}\left(u_{i}\right), \gamma_{2}\left(u_{i}\right)\right)$. Using the Faber-Krahn inequality in dimension $N-1$ we obtain

$$
\begin{aligned}
\int_{\left\{x_{1}=t\right\}} & u_{i}^{2} d \mathcal{H}^{N-1} \\
& \leq \frac{1}{c_{2}(t)}\left(\int_{\left\{x_{1}=t\right\}}\left|\nabla^{\prime} u_{i}\right|^{2} d \mathcal{H}^{N-1}+\beta \int_{J\left(u_{i}\right) \cap\left\{x_{1}=t\right\}}\left[\gamma_{1}\left(u_{i}\right)^{2}+\gamma_{2}\left(u_{i}\right)^{2}\right] d \mathcal{H}^{N-2}\right),
\end{aligned}
$$

where $\nabla^{\prime}$ stands for gradient with respect to $x_{2}, \ldots, x_{N}$, and $c_{2}(t)$ is given in (4.27). Claim (4.25) follows by combining (4.30) and (4.31).

Step 2. We claim that for a.e. $t \geq 0$ large enough

$$
\int_{\left\{x_{1}>t\right\}}|\nabla u|^{2} d x+\beta \sum_{i=1}^{k} \int_{J_{u_{i}} \cap\left\{x_{1}>t\right\}}\left[\gamma_{1}\left(u_{i}\right)^{2}+\gamma_{2}\left(u_{i}\right)^{2}\right] d \mathcal{H}^{N-1} \leq \frac{2 \delta(t)}{\sqrt{c_{2}(t)}},
$$

where $\delta(t)$ and $c_{2}(t)$ are given in (4.26) and (4.27) respectively.

In order to prove the claim, let us make use of inequality (4.19). By letting $\varphi$ approach the function

we obtain for every $i=1, \ldots, k$

$$
\varphi_{\varepsilon}(x):=\frac{\left(x_{1}-t\right)^{+}}{\varepsilon} \wedge 1
$$

$$
\begin{array}{r}
\int_{\mathbb{R}^{N}} \varphi_{\varepsilon}\left|\nabla u_{i}\right|^{2} d x+\frac{1}{\varepsilon} \int_{\left\{t<x_{1}<t+\varepsilon\right\}} u_{i} \nabla u_{i} \cdot e_{1} d x+\beta \int_{J_{u_{i}}} \varphi_{\varepsilon} \cdot\left[\gamma_{1}\left(u_{i}\right)^{2}+\gamma_{2}\left(u_{i}\right)^{2}\right] d \mathcal{H}^{N-1} \\
\leq \lambda_{k, \beta}^{m} \int_{\mathbb{R}^{N}} \varphi_{\varepsilon} \cdot u_{i}^{2} d x
\end{array}
$$


where $e_{1}$ is the horizontal vector $(1,0, \ldots, 0)$. Letting $\varepsilon \rightarrow 0^{+}$, we obtain for a.e. $t \in \mathbb{R}$

$$
\begin{aligned}
\int_{\left\{x_{1}>t\right\}}\left|\nabla u_{i}\right|^{2} d x+\beta & \int_{J_{u_{i}} \cap\left\{x_{1}>t\right\}}\left[\gamma_{1}\left(u_{i}\right)^{2}+\gamma_{2}\left(u_{i}\right)^{2}\right] d \mathcal{H}^{N-1} \\
& \leq \lambda_{k, \beta}^{m} \int_{\left\{x_{1}>t\right\}} u_{i}^{2} d x+\int_{\left\{x_{1}=t\right\}}\left|u_{i}\right|\left|\nabla u_{i}\right| d \mathcal{H}^{N-1} \\
& \leq \lambda_{k, \beta}^{m} \int_{\left\{x_{1}>t\right\}} u_{i}^{2} d x+\left(\int_{\left\{x_{1}=t\right\}}\left|u_{i}\right|^{2} d \mathcal{H}^{N-1} \int_{\left\{x_{1}=t\right\}}\left|\nabla u_{i}\right|^{2} d \mathcal{H}^{N-1}\right)^{1 / 2} .
\end{aligned}
$$

We estimate the last term using the Faber-Krahn inequality on the section of $u_{i}$ on $\left\{x_{1}=t\right\}$ as in the previous step: recalling (4.31), and replacing the tangential gradient with the full one we may write

$$
\begin{aligned}
\int_{\left\{x_{1}>t\right\}}\left|\nabla u_{i}\right|^{2} d x+\beta \int_{J_{u_{i}} \cap\left\{x_{1}>t\right\}}\left[\gamma_{1}\left(u_{i}\right)^{2}+\gamma_{2}\left(u_{i}\right)^{2}\right] d \mathcal{H}^{N-1} \leq \lambda_{k, \beta}^{m} \int_{\left\{x_{1}>t\right\}} u_{i}^{2} d x \\
+\frac{1}{\sqrt{c_{2}(t)}}\left[\int_{\left\{x_{1}=t\right\}}\left|\nabla u_{i}\right|^{2} d \mathcal{H}^{N-1}+\beta \int_{J_{u^{i}} \cap\left\{x_{1}=t\right\}}\left[\gamma_{1}\left(u_{i}\right)^{2}+\gamma_{2}\left(u_{i}\right)^{2}\right] d \mathcal{H}^{N-2}\right] .
\end{aligned}
$$

By employing a reflection with respect to $\left\{x_{1}=t\right\}$, and using the Faber-Krahn inequality of Theorem 3.3 for the positive and negative parts of $u_{i}$ we have

$$
\int_{\left\{x_{1}>t\right\}} u_{i}^{2} d x \leq \frac{1}{c_{1}(t)}\left[\int_{\left\{x_{1}>t\right\}}\left|\nabla u_{i}\right|^{2} d x+\beta \int_{J_{u_{i}} \cap\left\{x_{1}>t\right\}}\left[\gamma_{1}\left(u_{i}\right)^{2}+\gamma_{2}\left(u_{i}\right)^{2}\right] d \mathcal{H}^{N-1}\right],
$$

where $c_{1}(t)$ is given in (4.29). Since $c_{1}(t) \rightarrow+\infty$ as $t \rightarrow+\infty$, we deduce that the first term on the right hand side of (4.33) is negligible with respect to the left hand side. Summing over $i=1, \ldots, k$, we obtain claim (4.32).

Step 3. Let

$$
\sigma(t):=\left|\operatorname{supp}(u) \cap\left\{x_{1}=t\right\}\right| .
$$

We claim that there exist $C>0$ and $t_{0} \in \mathbb{R}$ such that for a.e. $t \geq t_{0}$

$$
\int_{t}^{+\infty} \sigma(\tau) d \tau \leq C|\sigma(t)|^{\frac{1}{N-1}}\left(1+|\sigma(t)|^{\frac{1}{N-1}}\right) \delta(t)
$$

and

$$
\int_{t}^{+\infty} \delta(\tau) d \tau \leq C|\sigma(t)|^{\frac{1}{2 N-2}}\left(1+|\sigma(t)|^{\frac{1}{2 N-2}}\right) \delta(t),
$$

where $\delta(t)$ is defined in (4.26).

In order to prove inequality (4.35), recall that

$$
m(t):=\left|\operatorname{supp}(u) \cap\left\{x_{1}>t\right\}\right|=\int_{t}^{+\infty} \sigma(\tau) d \tau .
$$
$t \in \mathbb{R}$

Using the optimality of $u$ together with the rescaling formula (4.16) we obtain that for a.e.

with

$$
R_{k, \beta}(u) \leq \frac{1}{\eta^{2}(t)} R_{k, \eta(t) \beta}\left(u 1_{\left\{x_{1}<t\right\}}\right)
$$

Since $\eta(t)>1$ we have

$$
\eta(t)^{N}:=\frac{|\operatorname{supp}(u)|}{|\operatorname{supp}(u)|-m(t)} .
$$

$$
R_{k, \beta}(u) \leq \frac{1}{\eta(t)} R_{k, \beta}\left(u 1_{\left\{x_{1}<t\right\}}\right)
$$


Using inequality (4.25) we obtain for a.e. $t \geq 0$ large enough

$$
\eta(t) R_{k, \beta}(u) \leq R_{k, \beta}(u)+\frac{2 \beta \delta(t)}{c_{2}(t)},
$$

which yields for $t$ large enough

$$
m(t) \leq \frac{C_{1} \delta(t)}{c_{2}(t)}
$$

for some $C_{1}>0$. By the very definition of $c_{2}(t)$ given in (4.27) and estimate (2.3) for the first Robin eigenvalue we have

$$
c_{2}(t) \geq \frac{\beta}{4|\sigma(t)|^{\frac{1}{N-1}}\left(1+\beta|\sigma(t)|^{\frac{1}{N-1}}\right)},
$$

so that inequality (4.35) follows.

Inequality (4.36) is a consequence of (4.32), in view of Fubini's theorem and of the coarea formula on the $\mathcal{H}^{N-1}$-countably rectifiable sets $J_{u_{i}}$ (see [1, Theorem 2.93]), and using again (4.37).

Step 4: Conclusion. Let us consider

$$
e(t):=\sigma(t)+\delta(t)
$$

and the absolutely continuous decreasing function $E:\left[t_{0},+\infty[\rightarrow] 0,+\infty[\right.$

$$
E(t):=\int_{t}^{+\infty} e(\tau) d \tau
$$

where $\sigma(t)$ and $\delta(t)$ are given in (4.34) and (4.26) respectively.

Summing inequalities (4.35) and (4.36), we deduce that for a.e. $t \geq t_{0}$

$$
\frac{E^{\prime}(t)}{\Phi^{-1}(E(t))} \leq-1
$$

where $\Phi:[0,+\infty[\rightarrow[0,+\infty[$ is the increasing function

$$
\Phi(s):=C_{1} s^{1+\frac{1}{2(N-1)}}\left[1+C_{2} s^{\frac{3}{2(N-1)}}\right]
$$

for suitable $C_{1}, C_{2}>0$. This inequality leads immediately to a contradiction. Indeed on one hand we have

$$
\left.\int_{t_{0}}^{+\infty} \frac{E^{\prime}(\tau)}{\Phi^{-1}(E(\tau))} d \tau=-\int_{0}^{E\left(t_{0}\right)} \frac{d s}{\Phi^{-1}(s)} \in\right]-\infty, 0[
$$

as for $s$ near zero we have

$$
\Phi^{-1}(s) \geq C_{3} s^{\left(1+\frac{1}{2(N-1)}\right)^{-1}}
$$

for some $C_{3}>0$. On the other, (4.38) entails

$$
\int_{t_{0}}^{+\infty} \frac{E^{\prime}(\tau)}{\Phi^{-1}(E(\tau))} d \tau=-\infty
$$

a contradiction. Note that this contradiction heavily relies on the fact that the support was supposed unbounded, so that the above integration in $t$ could be carried up to $+\infty$.

Lemma 4.7 (Splitting geometry). Let $u \in \mathcal{F}_{k}\left(\mathbb{R}^{N}\right)$ be such that

$$
u=u_{1}+u_{2}
$$

with supp $\left(u_{1}\right)$ and supp $\left(u_{2}\right)$ well separated (see subsection 2). The following items hold true.

(a) If $u_{1}=\left(v_{1}, 0\right)$ and $u_{2}=\left(0, v_{2}\right)$ with $v_{1} \in \mathcal{F}_{k_{1}}\left(\mathbb{R}^{N}\right)$ and $v_{2} \in \mathcal{F}_{k_{2}}\left(\mathbb{R}^{N}\right)$, we have

$$
R_{k, \beta}(u)=\max \left\{R_{k_{1}, \beta}\left(v_{1}\right), R_{k_{2}, \beta}\left(v_{2}\right)\right\} .
$$

(b) If $m_{i}:=\left|\operatorname{supp}\left(u_{i}\right)\right|>0$, then

$$
R_{k, \beta}(u) \geq \min _{j=0, \ldots, k} \max \left\{\lambda_{j, \beta}^{m_{1}}, \lambda_{k-j, \beta}^{m_{2}}\right\},
$$

with the convention $\lambda_{0, \beta}^{m}:=0$. 
Proof. Let us start with point (b), proving that

$$
R_{k, \beta}(u) \geq \max \left\{\lambda_{h_{1}, \beta}^{m_{1}}, \lambda_{h_{2}, \beta}^{m_{2}}\right\}
$$

for some $h_{1}+h_{2}=k$.

Let us consider the finite dimensional spaces $V\left(u_{1}\right)$ and $V\left(u_{2}\right)$ and their direct sum

$$
V:=V\left(u_{1}\right) \oplus V\left(u_{2}\right) .
$$

If we denote the associated dimensions by $k_{1}$ and $k_{2}$, we have clearly

$$
k \leq k_{1}+k_{2} \leq 2 k \text {. }
$$

Let us proceed by computing some kind of "discrete" eigenvalues on $V$ using the standard Rayleigh minimization process. More precisely, let $w_{1}$ be a minimizer of the quadratic energy

$$
Q(v):=\int_{\mathbb{R}^{N}}|\nabla v|^{2} d x+\beta \int_{J_{u}}\left[\gamma_{1}(v)^{2}+\gamma_{2}(v)^{2}\right] d \mathcal{H}^{N-1}
$$

on those functions $v \in V$ with $\|v\|_{L^{2}\left(\mathbb{R}^{N}\right)}=1$, and let $w_{j}$ be a minimizer of $Q$ on those functions in $V$, with unit $L^{2}$-norm, and $L^{2}$-orthogonal to $\left\{w_{1}, \ldots, w_{j-1}\right\}$. Let $\mu_{j}$ denote the associated "eigenvalues".

Using the inequality

$$
\frac{a+b}{c+d} \geq \min \left\{\frac{a}{c}, \frac{b}{d}\right\},
$$

we see that $w_{1}$, and recursively each $w_{j}$, belongs either to $V\left(u_{1}\right)$ or to $V\left(u_{2}\right)$. As a consequence

$$
\left\{\mu_{1}, \ldots, \mu_{k_{1}+k_{2}}\right\}=\left\{\eta_{1}, \ldots, \eta_{k_{1}}, \zeta_{1}, \ldots, \zeta_{k_{2}}\right\},
$$

where $\eta_{i}$ and $\zeta_{j}$ are the "discrete" eigenvalues associated to $V\left(u_{1}\right)$ and $V\left(u_{2}\right)$, obtained by the same variational procedure.

Assume

$$
\left\{\mu_{1}, \ldots, \mu_{k}\right\}=\left\{\eta_{1}, \ldots, \eta_{h_{1}}, \zeta_{1}, \ldots, \zeta_{h_{2}}\right\}
$$

with $h_{1}+h_{2}=k$. Since we can find in $V(u)$ an element which is $L^{2}$-orthogonal to $w_{1}, \ldots, w_{k-1}$ (since the orthogonality requires $k-1$ linear relations), we get that

$$
R_{k, \beta}(u) \geq \mu_{k}=\max \left\{\eta_{h_{1}}, \zeta_{h_{2}}\right\} .
$$

Since $\eta_{h_{1}} \geq \lambda_{h_{1}, \beta}^{m_{1}}$ and $\zeta_{h_{2}} \geq \lambda_{h_{2}, \beta}^{m_{2}}$, inequality (4.39) follows, and point (b) is proved.

Let us come back to point (a). Notice that by assumption

$$
V(u)=V_{k_{1}}\left(u_{1}\right) \oplus V_{k_{2}}\left(u_{2}\right) .
$$

Then we get that in the preceding argument $h_{1}=k_{1}$ and $h_{2}=k_{2}$, with $\eta_{h_{1}}=R_{k_{1}, \beta}\left(u_{1}\right)$, $\zeta_{h_{2}}=R_{k_{2}, \beta}\left(u_{2}\right)$, and $R_{k, \beta}(u)=\mu_{k}$. From (4.40) we deduce that point (a) holds true, and the proof is concluded.

4.3. Proof of Theorem 4.1. We are now ready to prove Theorem 4.1. Equality (4.5) is a consequence of Theorem 4.3 and of Remark 3.11. We can thus concentrate on the existence of minimizers with bounded support: this is obtained through a vanishing/dichotomy/compactness alternative together with an induction argument.

The result is known for $k=1$, being essentially the Faber-Krahn inequality of Theorem 3.3: minimizers are given by the first eigenfunctions of the ball, extended by zero to the entire space. We now proceed by induction, assuming that the result holds true for $k-1$, and proving it for $k$.

Let $\left(\Omega_{n}\right)_{n \in \mathbb{N}}$ be a "minimizing" sequence of Lipschitz domains, i.e., such that $\left|\Omega_{n}\right| \leq m$ and

$$
\lambda_{k, \beta}\left(\Omega_{n}\right) \rightarrow \lambda_{k, \beta}^{m}=\inf _{u \in \mathcal{F}_{k}^{m}\left(\mathbb{R}^{N}\right)} R_{k, \beta}(u) .
$$

Its existence is secured by relation (4.5). By Lemma 4.5, we know that

$$
u_{n}:=\left(u_{1}^{n}, \ldots, u_{k}^{n}\right) \in S B V_{ \pm}^{\frac{1}{2}}\left(\mathbb{R}^{N} ; \mathbb{R}^{k}\right)
$$


is a minimizing sequence for problem (4.3), where $\left\{u_{i}^{n}\right\}_{i=1, \ldots}$ is the orthonormal family of the first $k$ eigenfunctions, extended to zero outside $\Omega_{n}$. Notice that

$$
\int_{\mathbb{R}^{N}}\left|\nabla u_{n}\right|^{2} d x+\beta \int_{J_{u_{n}}}\left[\left|\gamma_{1}\left(u_{n}\right)\right|^{2}+\left|\gamma_{2}\left(u_{n}\right)\right|^{2}\right] d \mathcal{H}^{N-1} \leq k \lambda_{k, \beta}\left(\Omega_{n}\right) \leq C,
$$

where $C$ is independent of $n$.

Let us perform a concentration compactness alternative by considering the function

$$
\frac{1}{k}\left|u_{n}\right|^{2}=\frac{1}{k}\left[\left(u_{1}^{n}\right)^{2}+\cdots+\left(u_{k}^{n}\right)^{2}\right] \in L^{1}\left(\mathbb{R}^{N}\right) .
$$

For every $r>0$ let us consider the monotone increasing functions $\alpha_{n}:[0,+\infty[\rightarrow[0,+\infty[$

$$
\alpha_{n}(r):=\sup _{y \in \mathbb{R}^{N}} \int_{Q_{r}(y)} \frac{1}{k}\left|u_{n}\right|^{2} d x .
$$

Up to a subsequence, in view of Helly's selection theorem, we may assume that up to a subsequence

$$
\alpha_{n} \rightarrow \alpha \quad \text { pointwise on }[0,+\infty[
$$

for a suitable monotone increasing function $\alpha:[0,+\infty[\rightarrow[0,+\infty[$.

The following situations may occur.

(a) Vanishing: $\lim _{r \rightarrow+\infty} \alpha(r)=0$;

(b) Dichotomy: $\left.\lim _{r \rightarrow+\infty} \alpha(r)=\bar{\alpha} \in\right] 0,1[$;

(c) Compactness: $\lim _{r \rightarrow+\infty} \alpha(r)=1$.

Let us deal with the three cases separately.

Step 1: Vanishing cannot occur. Let us see that the vanishing case cannot occur. Thanks to (4.41), by [8, Lemma 4] applied to the positive and negative parts of the components of $u_{n}$, we can find $y_{n} \in \mathbb{R}^{N}$ such that

$$
\left|\operatorname{supp}\left(u_{n}\right) \cap Q_{1}\left(y_{n}\right)\right| \geq c>0,
$$

with $c$ independent of $n$. Since

$$
\lim _{n \rightarrow+\infty} \int_{Q_{1}\left(y_{n}\right)}\left|u_{n}\right|^{2} d x=0
$$

up to reducing the side of the cube, we may assume that for every $n \in \mathbb{N}$

$$
\mathcal{H}^{N-1}\left(J_{u_{n}} \cap \partial Q_{1}\left(y_{n}\right)\right)=0
$$

and

still preserving (4.42). Let us consider

$$
\lim _{n \rightarrow+\infty} \int_{\partial Q_{1}\left(y_{n}\right)}\left|u_{n}\right|^{2} d \mathcal{H}^{N-1}=0
$$

$$
w_{n}:=u_{n} 1_{\mathbb{R}^{N} \backslash Q_{1}\left(y_{n}\right)} \in S B V_{ \pm}^{\frac{1}{2}}\left(\mathbb{R}^{N} ; \mathbb{R}^{k}\right) .
$$

Notice that $\left|\operatorname{supp}\left(w_{n}\right)\right| \leq m-c$. Assume for a moment that $w_{n} \in \mathcal{F}_{k}^{m-c}\left(\mathbb{R}^{N}\right)$, that is that the associated vectorial space $V\left(w_{n}\right)$ has dimension $k$. A direct calculation shows that

$$
\liminf _{n \rightarrow+\infty} R_{k, \beta}\left(w_{n}\right) \leq \liminf _{n \rightarrow+\infty} R_{k, \beta}\left(u_{n}\right),
$$

so that

$$
\lambda_{k, \beta}^{m-c} \leq \lambda_{k, \beta}^{m} .
$$

This is against the monotonicity property (4.17).

Notice that if $V\left(w_{n}\right)$ has not dimension $k$, we can perturb the components by adding vanishing perturbations in $C_{c}^{\infty}\left(Q_{1}\left(y_{n}\right)\right)$ without affecting the previous considerations. We conclude that the vanishing case cannot occur.

Step 2: Existence in the dichotomy case. Let us assume that

$$
\lim _{r \rightarrow+\infty} Q(r)=\bar{\alpha}
$$


with $0<\bar{\alpha}<1$. Following [28, Lemma 1.1] , for every $0<\varepsilon<1$ there exist $R>0$ and $\left(x_{n}\right)_{n \in \mathbb{N}}$ such that, up to a subsequence, we can find $R_{n} \rightarrow+\infty$ with

$$
\left.\left|\int_{B_{R}\left(x_{n}\right)} \frac{1}{k}\right| u_{n}\right|^{2} d x-\bar{\alpha} \mid<\varepsilon \quad \text { and }\left.\quad\left|\int_{\mathbb{R}^{N} \backslash B_{R_{n}}\left(x_{n}\right)} \frac{1}{k}\right| u_{n}\right|^{2} d x-(1-\bar{\alpha}) \mid<\varepsilon .
$$

We can thus construct a cut-off function $\varphi_{n} \in C_{c}^{\infty}\left(\mathbb{R}^{N}\right)$ with $\left\|\nabla \varphi_{n}\right\|_{\infty}<\varepsilon, \varphi_{n}=1$ on $B_{R}\left(x_{n}\right)$ and on $\mathbb{R}^{N} \backslash B_{R_{n}}$, and such that $\operatorname{supp}\left(\varphi_{n}\right)=A_{n} \cup B_{n}$ with $B_{R}\left(x_{n}\right) \subseteq A_{n},\left(\mathbb{R}^{N} \backslash B_{R_{n}}\right) \subseteq B_{n}$ and $\operatorname{dist}\left(A_{n}, B_{n}\right) \rightarrow+\infty$.

Let us concentrate on the function $\varphi_{n} u_{n}$ which we may see as

$$
\varphi_{n} u_{n}=v_{n}+w_{n}
$$

with $\operatorname{supp}\left(v_{n}\right) \subseteq A_{n}$ and $\operatorname{supp}\left(w_{n}\right) \subseteq B_{n}$. We have

$$
\left\|u_{n}-v_{n}-w_{n}\right\|_{L^{2}\left(\mathbb{R}^{N}\right)}^{2}<2 k \varepsilon .
$$

Moreover, taking into account (4.41) and the properties of $\varphi_{n}$, we have

$$
\int_{\mathbb{R}^{N}}\left|\nabla v_{n}\right|^{2} d x+\int_{J_{v_{n}}}\left[\left|\gamma_{1}\left(v_{n}\right)\right|^{2}+\left|\gamma_{2}\left(v_{n}\right)\right|^{2}\right] d \mathcal{H}^{N-1} \leq C_{1}
$$

and

$$
\int_{\mathbb{R}^{N}}\left|\nabla w_{n}\right|^{2} d x+\int_{J_{w_{n}}}\left[\left|\gamma_{1}\left(w_{n}\right)\right|^{2}+\left|\gamma_{2}\left(w_{n}\right)\right|^{2}\right] d \mathcal{H}^{N-1} \leq C_{2}
$$

for some $C_{1}, C_{2}>0$ independent of $\varepsilon$.

Let

$$
\left|\operatorname{supp}\left(v_{n}\right)\right|=m_{n, 1} \quad \text { and } \quad\left|\operatorname{supp}\left(w_{n}\right)\right|=m_{n, 2} .
$$

We may assume up to a subsequence

$$
m_{n, 1} \rightarrow m_{1}^{\varepsilon} \quad \text { and } \quad m_{n, 2} \rightarrow m_{2}^{\varepsilon}
$$

with $m_{1}^{\varepsilon}+m_{2}^{\varepsilon} \leq m$. In view of (4.45), (4.46), (4.43) and of the Faber-Krahn inequality of Theorem 3.3 (applied to positive and negative parts of $v_{n}$ and $w_{n}$ ), we deduce that $m_{1}^{\varepsilon}>\eta$ and $m_{2}^{\varepsilon}>\eta$ for some $\eta>0$ independent of $\varepsilon$.

It is not restrictive to assume $\varphi_{n} u_{n} \in \mathcal{F}_{k}\left(\mathbb{R}^{N}\right)$ : indeed, the associated vectorial space $V\left(\varphi_{n} u_{n}\right)$ can be made $k$-dimensional up to adding vanishing smooth functions with small compact support, without affecting the asymptotic behaviour outlined above.

Taking into account (4.41) and (4.44) we get

$$
e(\varepsilon)+R_{k, \beta}\left(u_{n}\right) \geq R_{k, \beta}\left(v_{n}+w_{n}\right),
$$

where $e(\varepsilon) \rightarrow 0$ as $\varepsilon \rightarrow 0^{+}$.

Up to a subsequence we obtain thanks to Lemma 4.7

$$
e(\varepsilon)+R_{k, \beta}\left(u_{n}\right) \geq \min _{h=0, \ldots, k} \max \left\{\lambda_{h, \beta}^{m_{n, 1}}, \lambda_{k-h, \beta}^{m_{n, 2}}\right\},
$$

with the convention $\lambda_{0, \beta}^{m}:=0$. Passing to the limit for $n \rightarrow+\infty$ and $\varepsilon \rightarrow 0^{+}$, and using a rescaling argument, we find $m_{1}>0, m_{2}>0$ with $m_{1}+m_{2}=m$ and such that

$$
\lambda_{k, \beta}^{m} \geq \min _{h=0, \ldots, k} \max \left\{\lambda_{h, \beta}^{m_{1}}, \lambda_{k-h, \beta}^{m_{2}}\right\}=\max \left\{\lambda_{j, \beta}^{m_{1}}, \lambda_{k-j, \beta}^{m_{2}}\right\}
$$

for some $0 \leq j \leq k$. We can exclude the cases $j=0$ and $j=k$ : indeed we would get $\lambda_{k, \beta}^{m} \geq \lambda_{k, \beta}^{m_{1}}$ or $\lambda_{k, \beta}^{m} \geq \lambda_{k, \beta}^{m_{2}}$ against the monotonicity property (4.17).

Using the inductive hypothesis, let $v \in \mathcal{F}_{j}^{m_{1}}\left(\mathbb{R}^{N}\right)$ and $w \in \mathcal{F}_{k-j}^{m_{2}}\left(\mathbb{R}^{N}\right)$ be minimizers for $\lambda_{j, \beta}^{m_{1}}$ and $\lambda_{k-j, \beta}^{m_{2}}$ with bounded support, which up to a translation, we may assume to be well separated. Let us consider the function

$$
u:=(v, 0)+(0, w) \in \mathcal{F}_{k}^{m}\left(\mathbb{R}^{N}\right)
$$

By Lemma 4.7 we deduce

$$
R_{k, \beta}(u)=\max \left\{R_{j, \beta}(v), R_{k-j, \beta}(w)\right\}=\max \left\{\lambda_{j, \beta}^{m_{1}}, \lambda_{k-j, \beta}^{m_{2}}\right\} .
$$


Thanks to (4.47) we deduce that $u$ is a minimizer of problem (4.3) with bounded support, and the step is concluded.

Step 3: Existence in the compact case. Let us assume that $\lim _{r \rightarrow+\infty} \alpha(r)=1$. There exists $\left(x_{n}\right)_{n \in \mathbb{N}}$ such that for every $\varepsilon>0$ there exist $R>0$ with

$$
\left.\left|\int_{Q_{R}\left(x_{n}\right)} \frac{1}{k}\right| u_{n}\right|^{2} d x-1 \mid<\varepsilon .
$$

We may assume $x_{n}=0$. Up to a subsequence, thanks to (4.41) and to Theorem 3.6 (recall that $\frac{1}{k} \int_{\mathbb{R}^{N}}\left|u_{n}\right|^{2} d x=1$ ) we have

$$
u_{n} \rightarrow u \quad \text { strongly in } L_{l o c}^{2}\left(\mathbb{R}^{N} ; \mathbb{R}^{k}\right)
$$

for some $u \in L^{2}\left(\mathbb{R}^{N} ; \mathbb{R}^{k}\right)$. Since by (4.48) we get $\|u\|_{L^{2}}=1$, we deduce

$$
u_{n} \rightarrow u \quad \text { strongly in } L^{2}\left(\mathbb{R}^{N} ; \mathbb{R}^{k}\right) .
$$

We can now apply Lemma 4.5 and Theorem 4.6: we deduce that $u \in \mathcal{F}_{k}^{m}\left(\mathbb{R}^{N}\right)$ is a minimizer of problem (4.3) with bounded support. The proof is now concluded.

\section{Further REMARKS AND OPEN QUESTIONS}

More regularity of optimal geometries. Obtaining more qualitative results on the optimal solution is a challenging problem. In particular, just to prove that the optimal solution corresponds to an open set, with a topological boundary of finite $\mathcal{H}^{N-1}$-measure, would be a very interesting result. Some techniques have already been developed in $[9,15]$ to handle energy type problems, as for instance corresponding to the first eigenvalue. A nontrivial such a problem is to replace the full space $\mathbb{R}^{N}$ (where the minimum for $\lambda_{1, \beta}$ is the ball) by a bounded, Lipschitz, open set $D$, i.e. to solve

$$
\min \left\{\lambda_{1, \beta}(\Omega): \Omega \subset D,|\Omega|=m\right\} .
$$

If $D$ does not contain a ball of volume $m$, one could prove that an optimal set exists, it is open and has a topological boundary of finite $\mathcal{H}^{N-1}$-measure. Moreover, it is likely that the regularity techniques developed in [15] could be extended to this problem.

Nevertheless, in order to get qualitative information for higher order eigenvalues, the crucial difficulty comes from the fact that $\lambda_{k}$ is itself a critical point. In the case of Dirichlet boundary conditions, some properties of the corresponding optimal sets have been obtained in [10] using the tool of shape subsolutions. The key idea was to transform optimality of the critical point $\lambda_{k}$, in some suboptimality of the torsion energy. This was a consequence of the possibility to control the variation of the $k$-th eigenvalue by the variation of the torsion energy for inner perturbations of a domain. In the case of Robin boundary conditions, this strategy seems to fail. In fact, if such a control would be available, it would also give an ordering of the shape derivatives of the $k$-th eigenvalue, and the torsion energy, respectively. Looking to the both shape gradients, this seems to be false, as their expression contains both curvature terms multiplied by the state functions and tangential parts of their gradients. These terms are difficult, if not impossible, to compare.

About simplicity of eigenvalues at optimal sets. For Dirichlet boundary conditions, it is a longstanding conjecture to prove that the $k$-th eigenvalue on an optimal set is multiple, precisely equal to $\lambda_{k-1}$. For Robin boundary conditions, the same property is expected to occur. We can even justify it, at least for solutions $\Omega$ of (1.1) which are open sets. Assume for contradiction, that $\Omega$ is such a solution for (1.1), and that $\lambda_{k, \beta}(\Omega)>\lambda_{k-1, \beta}(\Omega)$. One can moreover assume that $\Omega$ is connected, otherwise we contradict the statement for some $1<k^{\prime}<k$. Necessarily, the $k$-th eigenfunction $u_{k}$ has a nontrivial nodal line $\mathcal{N}_{k}=\left\{u_{k}=0\right\} \cap \Omega$. There exists a point $x_{0} \in \mathcal{N}_{k}$ such that $\mathcal{N}_{k}$ is an analytic surface around $x_{0}$. We remove a set $S$ from this neigbourhood which is analytic surface of dimension $(N-1)$ and has a small capacity. The size in terms of capacity can be chosen such that the $(k-1)$ eigenvalue of the mixed problem with Robin boundary conditions 
on $\partial \Omega$ and Dirichlet boundary conditions on $S$ is still lower than $\lambda_{k, \beta}(\Omega)$. For this mixed problem, we notice

$$
\lambda_{k-1}^{\text {mixed }}<\lambda_{k, \beta}(\Omega)=\lambda_{k}^{\text {mixed }} .
$$

The last equality is true, since $u_{k}$ is already vanishing on $S$, hence is an eigenfunction of the mixed problem.

On the other hand, by monotonicity of spaces $\left\{u \in H^{1}(\Omega \backslash S): u=0\right.$ on $\left.S\right\} \subset H^{1}(\Omega \backslash S)$, one can order

$$
\lambda_{k, \beta}(\Omega \backslash S) \leq \lambda_{k}^{\text {mixed }}=\lambda_{k, \beta}(\Omega) .
$$

Since the inequality above can not be strict, as $\Omega$ is an optimal set, we have to have equality, and in this case the eigenfunction $u_{k}$ of $\Omega$ is also an eigenfunction for $\lambda_{k, \beta}(\Omega \backslash S)$. From the Robin boundary condition on $S$, this means that $\nabla u_{k}$ has to vanish on $S$. This is in contradiction with the Hopf principle.

\section{REFERENCES}

[1] L. Ambrosio, N. Fusco, and D. Pallara. Functions of bounded variation and free discontinuity problems. Oxford Mathematical Monographs. The Clarendon Press Oxford University Press, New York, 2000.

[2] Pedro R. S. Antunes and Pedro Freitas. Numerical optimization of low eigenvalues of the Dirichlet and Neumann Laplacians. J. Optim. Theory Appl., 154(1):235-257, 2012.

[3] Pedro R. S. Antunes, Pedro Freitas, and David Krejčiřík. Bounds and extremal domains for Robin eigenvalues with negative boundary parameter. Adv. Calc. Var., 10(4):357-379, 2017.

[4] Pedro Ricardo Simão Antunes, Pedro Freitas, and James Bernard Kennedy. Asymptotic behaviour and numerical approximation of optimal eigenvalues of the Robin Laplacian. ESAIM Control Optim. Calc. Var., 19(2):438-459, 2013.

[5] M.-H. Bossel. Membranes élastiquement liées: extension du théorème de Rayleigh-Faber-Krahn et de l'inégalité de Cheeger. C. R. Acad. Sci. Paris Sér. I Math., 302(1):47-50, 1986.

[6] Marie-Hélène Bossel. Membranes élastiquement liées inhomogènes ou sur une surface: une nouvelle extension du théorème isopérimétrique de Rayleigh-Faber-Krahn. Z. Angew. Math. Phys., 39(5):733-742, 1988.

[7] A. Braides. Approximation of free-discontinuity problems, volume 1694 of Lecture Notes in Mathematics. Springer-Verlag, Berlin, 1998.

[8] D. Bucur and A. Giacomini. A variational approach to the isoperimetric inequality for the Robin eigenvalue problem. Arch. Ration. Mech. Anal., 198(3):927-961, 2010.

[9] D. Bucur and A. Giacomini. Shape optimization problems with Robin conditions on the free boundary. Ann. Inst. H. Poincaré Anal. Non Linéaire, 33(6):1539-1568, 2016.

[10] Dorin Bucur. Minimization of the $k$-th eigenvalue of the Dirichlet Laplacian. Arch. Ration. Mech. Anal., 206(3):1073-1083, 2012.

[11] Dorin Bucur and Simone Cito. Geometric control of the robin laplacian eigenvalues: the case of negative boundary parameter. Preprint Cvgmt, 2018.

[12] Dorin Bucur and Daniel Daners. An alternative approach to the Faber-Krahn inequality for Robin problems. Calc. Var. Partial Differential Equations, 37(1-2):75-86, 2010.

[13] Dorin Bucur and Alessandro Giacomini. Faber-Krahn inequalities for the Robin-Laplacian: a free discontinuity approach. Arch. Ration. Mech. Anal., 218(2):757-824, 2015.

[14] G. Buttazzo and G. Dal Maso. An existence result for a class of shape optimization problems. Arch. Rational Mech. Anal., 122(2):183-195, 1993.

[15] Luis A. Caffarelli and Dennis Kriventsov. A free boundary problem related to thermal insulation. Comm. Partial Differential Equations, 41(7):1149-1182, 2016.

[16] G. Cortesani and R. Toader. A density result in SBV with respect to non-isotropic energies. Nonlinear Anal., 38(5, Ser. B: Real World Appl.):585-604, 1999.

[17] Gianni Dal Maso, Gilles A. Francfort, and Rodica Toader. Quasistatic crack growth in nonlinear elasticity. Arch. Ration. Mech. Anal., 176(2):165-225, 2005.

[18] D. Daners. A Faber-Krahn inequality for Robin problems in any space dimension. Math. Ann., 335(4):767-785, 2006.

[19] D. Daners and J. Kennedy. Uniqueness in the Faber-Krahn inequality for Robin problems. SIAM J. Math. Anal., 39(4):1191-1207, 2007/08.

[20] Guido De Philippis and Bozhidar Velichkov. Existence and regularity of minimizers for some spectral functionals with perimeter constraint. Appl. Math. Optim., 69(2):199-231, 2014.

[21] L. C. Evans and R. F. Gariepy. Measure theory and fine properties of functions. Studies in Advanced Mathematics. CRC Press, Boca Raton, FL, 1992.

[22] Gilles A. Francfort and Christopher J. Larsen. Existence and convergence for quasi-static evolution in brittle fracture. Comm. Pure Appl. Math., 56(10):1465-1500, 2003.

[23] T. Giorgi and R. G. Smits. Monotonicity results for the principal eigenvalue of the generalized Robin problem. Illinois J. Math., 49(4):1133-1143 (electronic), 2005. 
[24] Antoine Henrot, editor. Shape optimization and spectral theory. De Gruyter Open, Warsaw, 2017.

[25] J. B. Kennedy. On the isoperimetric problem for the higher eigenvalues of the Robin and Wentzell Laplacians. Z. Angew. Math. Phys., 61(5):781-792, 2010.

[26] Hynek Kovařík. On the lowest eigenvalue of Laplace operators with mixed boundary conditions. J. Geom. Anal., 24(3):1509-1525, 2014.

[27] Dennis Kriventsov. A free boundary problem related to thermal insulation: Flat implies smooth. Preprint, (https://arxiv.org/abs/1511.05949), 2015.

[28] P.-L. Lions. The concentration-compactness principle in the calculus of variations. The locally compact case. I. Ann. Inst. H. Poincaré Anal. Non Linéaire, 1(2):109-145, 1984.

(Dorin Bucur) Institut Universitaire de France and Laboratoire de Mathématiques, CNRS UMR 5127, Université Savoie Mont Blanc, Campus Scientifique, 73376 Le-Bourget-Du-Lac, France

E-mail address, D. Bucur: dorin.bucur@univ-savoie.fr

(Alessandro Giacomini) DiCAtam, Sezione di Matematica, Università degli Studi di Brescia, Via Branze 43, 25133 Brescia, ItAly

E-mail address, A. Giacomini: alessandro.giacomini@unibs.it 